\title{
Teleportation, Braid Group and Temperley-Lieb Algebra
}

\author{
Yong Zhang ${ }^{1}$ \\ Institute of Theoretical Physics, Chinese Academy of Sciences \\ P. O. Box 2735, Beijing 100080, P. R. China
}

\begin{abstract}
In the paper, we describe the teleportation from the viewpoints of the braid group and Temperley-Lieb algebra. We propose the virtual braid teleportation which exploits the teleportation swapping and identifies unitary braid representations with universal quantum gates, and further suggest the braid teleportation which is explained in terms of the crossed measurement and the state model of knot theory. In view of the diagrammatic representation for the Temperley-Lieb algebra, we devise diagrammatic rules for an algebraic expression and apply them to various topics around the teleportation: the transfer operator and acausality problem; teleportation and measurement; all tight teleportation and dense coding schemes; the Temperley-Lieb algebra and maximally entangled states; entanglement swapping; teleportation and topological quantum computing; teleportation and the Brauer algebra; multipartite entanglements. All examples clearly suggest the Temperley-Lieb algebra under local unitary transformations to be the algebraic structure underlying the teleportation. We show the teleportation configuration to be a fundamental element in the diagrammatic representation for the Brauer algebra and suggest a new equivalent approach to the teleportation in terms of the swap gate and Bell measurement. To propose our diagrammatic rules to be a natural diagrammatic language for the teleportation, we compare it with the other two known approaches to the quantum information flow: the teleportation topology and strongly compact closed category, and make essential differences among them as clear as possible.
\end{abstract}

Key Words: Teleportation, Braid group, Temperley-Lieb algebra

PACS numbers: 03.65.Ud, 02.10.Kn, 03.67.Lx

\footnotetext{
${ }^{1}$ yong@itp.ac.cn
} 


\section{Introduction}

Quantum entanglements [1] play the key roles in quantum information 2] and have been widely exploited in quantum algorithms [3, 4, quantum cryptography [5, 6] and quantum teleportation [7. On the other hand, topological entanglements [8] denote topological configurations like links or knots which are closures of braids. There are natural similarities between quantum entanglements and topological entanglements. As a unitary braid has the power of detecting knots or links, it is able to transform a separate quantum state into an entangled one. Hence a nontrivial unitary braid representation can be identified with a universal quantum gate [9]. Recently, a series of papers have been published on the application of knot theory to quantum information, see 10, 11, 12 for universal quantum gates and unitary solutions of the Yang-Baxter equation [13, 14]; see [15, 16, 17] for quantum topology and quantum computation; see [18, 19] for quantum entanglements and topological entanglements.

Especially, Kauffman's work on the teleportation topology [10, 20] motivates our tour of revisiting in a diagrammatic approach all tight teleportation and dense coding schemes in Werner's paper 21. Under the project of setting up a bridge between knot theory and quantum information, the joint paper with Kauffman and Werner 22. makes a survey of diagrammatic tensor calculus and matrix representations and explores topological and algebraic structures underlying multipartite entanglements and then applies them to the realignment criteria of multipartite entanglements, while the present one focuses on the problem of how to study the teleportation from the viewpoints of the braid group and Temperley-Lieb algebra [23.

The teleportation is an amazing procedure of sending a message from Charlie to Bob under the help of Alice. Alice shares a maximally entangled state with Bob but makes the entangling measurement on the composite system between Charlie and her and then informs results of her measurement to Bob who will know what Charlie wants to pass onto him according to the protocol between Alice and him. The transformation matrix between the Bell states and product basis is found out to satisfy the braid relation and so stimulates us to study the teleportation at the level of the braid group. The teleportation equation in terms of braids at least has two types of interpretations, and we propose both the virtual braid teleportation and the braid teleportation.

The virtual braid group [24] is generated by virtual crossings and braids. A virtual crossing acts like permutation $P$ in the teleportation swapping $(P \otimes I d)(I d \otimes$ $P)$ or $(I d \otimes P)(P \otimes I d), I d$ for identity, while a unitary braid transforms a separate state into an entangled one as a universal quantum gate does. We propose the teleportation swapping to be the way of performing the teleportation in terms of the swap gate $P$. The virtual crossing relation for defining the virtual braid group will be explained as a kind of formulation of the teleporation equation. Underlying the virtual braid teleportation, the Brauer algebra [25] or the virtual TemperleyLieb algebra [22], has the teleportation configuration playing the fundamental roles in its diagrammatic representation.

The braid teleportation is proposed because we formulate the teleportation equa- 
tion in terms of the Bell matrix and have to present a sort of braid interpretation for it. We explain the braid teleportation in the sense of the crossed measurement 26. But there is an ambiguity in the braid teleportation: We can not fix the braid configuration related to the braid teleportation. In the state model of knot theory [8], a braid representation is constructed in terms of the Temperley-Lieb algebra which is the right one responsible for the teleportation, as leads to the observation that the braid teleportation consists of teleportation terms and non-teleportation terms. Therefore, we spend our main efforts in making clear complexities between the Temperley-Lieb algebra and teleportation.

The maximally entangled state shared by Alice and Bob is found to be a projector and form the representation of the Temperley-Lieb algebra. In view of the diagrammatic representation for the Temperley-Lieb algebra, we are inspired to deal with various topics on the teleportation in a diagrammatic approach. We devise our diagrammatic rules to describe a regular algebraic expression of quantum mechanics in terms of a diagram. A maximally entangled vector is denoted by the configuration of cup (cap) and a local unitary transformation is denoted by a solid point or a small circle on a branch of the cup (cap). A projector, trace and transfer operator each correspond to a specified combination of a cup with a cap. An operator, Dirac bra and Dirac ket are denoted by a middle solid point, a top solid point and a bottom solid point, respectively. Note that our diagrammatic rules are invented on the purpose of describing the teleportation instead of dealing with all subjects of quantum mechanics.

Besides its standard description [7, the teleportation has been viewed in terms of the transfer operator [27, from the point of quantum measurement [26] and in the tight teleportation scheme [21], respectively. We will revisit each of them in our diagrammatic approach. The transfer operator has a configuration involving a top cap and a bottom cup in which the teleportation appears to be a kind of the flow of quantum information and the so called acausality problem arises that the state preparation seems to happen after the measurement. As the state preparation is also a result of the measurement, the entire teleportation process has a description in the language of quantum measurement. Its diagrammatic realization exploits a combined top cup with a bottom cap to denote a projector representing measurement, as easily unifies the teleportation schemes of discrete and continuous variables into a picture.

In the tight teleportation scheme [21], Alice, Bob and Charlie have the Hilbert spaces of the same dimension and the teleportation is exactly encoded in an algebraic equation. We present all tight teleportation (dense coding) schemes in a diagrammatic approach, which sheds a light on our observation of the TemperleyLieb algebra underlying the teleportation. Importantly, both the transfer operator and the viewpoint of quantum measurement are contained in the diagrammatic description for the tight teleportation scheme. With cups and caps, the axioms of the Temperley-Lieb algebra are easily verified in a diagrammatic way and so the teleportation configuration is an element of the diagrammatic representation for the Temperley-Lieb algebra. For convenience, we call the Temperley-Lieb category for 
the Temperley-Lieb algebra under local unitary transformations, which represents the configurations consisting of cups, caps and solid points or small circles in the diagrammatic sense.

As the teleportation has been exploited in topics outside itself, we apply our diagrammatic rules to the entanglement swapping [28] and quantum computing [29]. The entanglement swapping is a way of yielding an entangled state between two systems which never met before and so have no physical interactions. Its diagrammatic description, an element of the Temperley-Lieb category, easily derives an algebraic equation for the entanglement swapping and also has a similar tight picture like the tight teleportation scheme. The teleportation provides an economic way of performing quantum computation. We find corresponding configurations in the Temperley-Lieb category for the unitary braid gate, swap gate and CNOT gate and then argue a possible quantum simulation of topological quantum computing in terms of devices for the teleportation. Additionally, we discuss how to represent multipartite entangled states with our diagrammatic rules. After various examples for the application of our diagrammatic rules have been presented, we come to the most important point of our paper and clearly states that the teleportation configuration is a fundamental ingredient in the diagrammatic description for the Brauer algebra [25] and can be performed in terms of the swap gate and Bell measurment.

Before our diagrammatic rules for the teleportation, there are two well known approaches to the quantum information flow: Kauffman's teleportation topology [10. 20] and the categorical theory mainly considered by Coecke [30. They are compared with our diagrammatical approaches to stress conceptual differences in both physics and mathematics among them and make it clear that our proposal catches essential points of the teleportation and is a natural diagrammatic language for it. The teleportation topology describes the teleportation also in terms of cups and caps but emphasizes the topological condition which is not satisfied in our case. We represent the quantum information flow by the transfer operator instead of identity in the teleportation topology. The category theory describes the quantum information flow by compositions of maps and identifies it with the strongly compact closed category. There exists essential conceptual differences from ours. We regard the quantum information flow as a part of the entire teleportation process and represent a projector in terms of a combined top cup with a bottom cap instead of a single cup or cap chosen by the category theory. Therefore, we propose the Temperley-Lieb algebra or Brauer algebra for the mathematical description of the teleportation.

The plans of the paper are organized as follows. The section 2 introduces the standard version of the teleportation, rewrites the teleportation equation in terms of the braid representation, and then proposes both the virtual braid teleportation and the braid teleportation. The section 3 presents our rules for drawing diagrams and then applies them to the transfer operator and acausality problem; teleportation and measurement; all tight teleportation and dense coding schemes; the Temperley-Lieb algebra and maximally entangled states; entanglement swapping; teleportation and topological quantum computing; teleportation and the Brauer algebra; multipartite 
entanglements. The section 4 sketches the teleportation topology and categorical approach in order to compare them with our methods. The section 5 is on concluding remarks.

\section{Teleportation and braid group}

We revisit the standard description of the teleportation [7] and rewrite the teleportation equation in terms of the Bell matrix which is proved to form the braid representation. We propose the teleportation swapping and explain the teleportation in the context of the virtual braid group. We further suggest the braid teleportation and present the interpretations in terms of both the crossed measurement [26] and state model of knot theory [8].

\subsection{Notations for the teleportation equation}

We fix notations for the Pauli matrices, qubit and the Bell states. The Pauli matrices $\sigma_{1}, \sigma_{2}$ and $\sigma_{3}$ have the conventional forms,

$$
\sigma_{1}=\left(\begin{array}{cc}
0 & 1 \\
1 & 0
\end{array}\right), \quad \sigma_{2}=\left(\begin{array}{cc}
0 & -i \\
i & 0
\end{array}\right), \quad \sigma_{3}=\left(\begin{array}{cc}
1 & 0 \\
0 & -1
\end{array}\right),
$$

and the quantum state $|0\rangle$ and $|1\rangle$ are identified with the matrices,

$$
|0\rangle=\left(\begin{array}{l}
1 \\
0
\end{array}\right), \quad|1\rangle=\left(\begin{array}{l}
0 \\
1
\end{array}\right)
$$

so that we have the following useful formulas,

$$
\sigma_{1}\left(\begin{array}{l}
a \\
b
\end{array}\right)=\left(\begin{array}{l}
b \\
a
\end{array}\right),-i \sigma_{2}\left(\begin{array}{l}
a \\
b
\end{array}\right)=\left(\begin{array}{c}
-b \\
a
\end{array}\right), \sigma_{3}\left(\begin{array}{c}
a \\
b
\end{array}\right)=\left(\begin{array}{c}
a \\
-b
\end{array}\right) .
$$

The four product basis of two-fold tensor products are chosen to be

$$
|00\rangle=\left(\begin{array}{l}
1 \\
0 \\
0 \\
0
\end{array}\right),|01\rangle=\left(\begin{array}{l}
0 \\
1 \\
0 \\
0
\end{array}\right),|10\rangle=\left(\begin{array}{l}
0 \\
0 \\
1 \\
0
\end{array}\right),|11\rangle=\left(\begin{array}{l}
0 \\
0 \\
0 \\
1
\end{array}\right)
$$

which fixes the rules for calculating the tensor product of matrices, i.e., embedding the right matrix into the left one. With the product basis $|i j\rangle, i, j=0,1$, the four mutually orthogonal Bell states have the forms,

$$
\begin{aligned}
\left|\phi^{+}\right\rangle & =\frac{1}{\sqrt{2}}(|00\rangle+|11\rangle), & \left|\phi^{-}\right\rangle & =\frac{1}{\sqrt{2}}(|00\rangle-|11\rangle), \\
\left|\psi^{+}\right\rangle & =\frac{1}{\sqrt{2}}(|01\rangle+|10\rangle), & \left|\psi^{-}\right\rangle & =\frac{1}{\sqrt{2}}(|01\rangle-|10\rangle),
\end{aligned}
$$


which derives the product basis $|i j\rangle$ in terms of the Bell states,

$$
\begin{array}{rlrl}
|00\rangle & =\frac{1}{\sqrt{2}}\left(\left|\phi^{+}\right\rangle+\left|\phi^{-}\right\rangle\right), & |01\rangle & =\frac{1}{\sqrt{2}}\left(\left|\psi^{+}\right\rangle+\left|\psi^{-}\right\rangle\right), \\
|10\rangle & =\frac{1}{\sqrt{2}}\left(\left|\psi^{+}\right\rangle-\left|\psi^{-}\right\rangle\right), & |11\rangle=\frac{1}{\sqrt{2}}\left(\left|\phi^{+}\right\rangle-\left|\phi^{-}\right\rangle\right) .
\end{array}
$$

The Bell states are transformed to each other under local unitary transformations,

$$
\begin{aligned}
& \left|\phi^{-}\right\rangle=\left(\mathbb{1}_{2} \otimes \sigma_{3}\right)\left|\phi^{+}\right\rangle=\left(\sigma_{3} \otimes \mathbb{1}_{2}\right)\left|\phi^{+}\right\rangle, \\
& \left|\psi^{+}\right\rangle=\left(\mathbb{1}_{2} \otimes \sigma_{1}\right)\left|\phi^{+}\right\rangle=\left(\sigma_{1} \otimes \mathbb{1}_{2}\right)\left|\phi^{+}\right\rangle, \\
& \left|\psi^{-}\right\rangle=\left(\mathbb{1}_{2} \otimes-i \sigma_{2}\right)\left|\phi^{+}\right\rangle=\left(i \sigma_{2} \otimes \mathbb{1}_{2}\right)\left|\phi^{+}\right\rangle,
\end{aligned}
$$

where $\mathbb{1}_{2}$ denotes $2 \times 2$ unit matrix and so $\mathbb{1}_{d}$ for $d \times d$ unit matrix.

The teleportation is an application of both quantum entanglement and quantum measurement and transports a unknown quantum state $|\psi\rangle_{C}=(a|0\rangle+b|1\rangle)_{C}$ from Charlie to Bob without classical communication between them. Let Alice and Bob share the Bell state $\left|\phi^{+}\right\rangle_{A B}$, a maximally entangled state which works as Alice is far away from Bob. Do the following calculation, also see [27,

$$
\begin{aligned}
& |\psi\rangle_{C}\left|\phi^{+}\right\rangle_{A B}=\frac{1}{\sqrt{2}}(a|0\rangle+b|1\rangle)_{C}(|00\rangle+|11\rangle)_{A B} \\
= & \frac{1}{2} a\left(\left|\phi^{+}\right\rangle+\left|\phi^{-}\right\rangle\right)_{C A}|0\rangle_{B}+\frac{1}{2} a\left(\left|\psi^{+}\right\rangle+\left|\psi^{-}\right\rangle\right)_{C A}|1\rangle_{B} \\
& +\frac{1}{2} b\left(\left|\psi^{+}\right\rangle-\left|\psi^{-}\right\rangle\right)_{C A}|0\rangle_{B}+\frac{1}{2} b\left(\left|\phi^{+}\right\rangle-\left|\phi^{-}\right\rangle\right)_{C A}|1\rangle_{B} \\
= & \frac{1}{2}\left(\left|\phi^{+}\right\rangle_{C A}|\psi\rangle_{B}+\left|\phi^{-}\right\rangle_{C A} \sigma_{3}|\psi\rangle_{B}+\left|\psi^{+}\right\rangle_{C A} \sigma_{1}|\psi\rangle_{B}+\left|\psi^{-}\right\rangle_{C A}\left(-i \sigma_{2}\right)|\psi\rangle_{B}\right)(8)
\end{aligned}
$$

which is called the teleportation equation in our paper and tells how to teleport the qubit $|\psi\rangle_{C}$ from Charlie to Bob with the help of Alice. Alice performs the Bell measurement in the composite system of Charlie and her and will have four kinds of outcomes. When Alice detects the Bell state $\left|\phi^{+}\right\rangle_{C A}$ and informs Bob about it through a classical channel, Bob will know that he has the quantum state $|\psi\rangle_{B}$ which Charlie wants to send to him. Similarly, when Alice gets the Bell states $\left|\phi^{-}\right\rangle_{C A}$ or $\left|\psi^{+}\right\rangle_{C A}$ or $\left|\psi^{-}\right\rangle_{C A}$, Bob will apply the local unitary transformations $\sigma_{3}$ or $\sigma_{1}$ or $i \sigma_{2}$ on the quantum state that he has in order to obtain the quantum state $|\psi\rangle_{B}$.

Note that the quantum state $|\psi\rangle_{C}$ Charlie has is destroyed via Alice's Bell measurement. We have to admit the teleportation to be a sort of magic and remain mystery until now for who are used to classical physical laws and classical communication. It is so simple for Bob to get a perfect copy of the quantum state that Charlie has although they do not have classical communication or interaction between them. Only fundamental laws of quantum mechanics and a little linear algebra are involved in the standard description of the teleportation. However, we believe that there exist beautiful mathematical structures underlying the teleportation. In the paper, we will make the fact clear that the teleportation configuration has been already 
in the diagrammatic description for the braid group, Temperley-Lieb algebra and Brauer algebra which were found and accepted by the mathematical community far before the proposal of the teleportation. Hence our main statement of this paper is addressed in the way: as we understand the teleportation in the framework of the Temperley-Lieb algebra, it will be natural and clear as it is. Most importantly, we think that the entire quantum information theory needs to be described in a powerful mathematical framework which is not linear algebra or Hilbert space for standard quantum mechanics.

\subsection{Teleportation equation in terms of Bell matrix}

Let introduce the so called Bell matrix [10, 11, 31, denoted by $B=\left(B_{i j, l m}\right)$, $i, j, l, m=0,1$. The $B$ matrix and its inverse $B^{-1}$ or transpose $B^{T}$ are given by

$$
B=\frac{1}{\sqrt{2}}\left(\begin{array}{cccc}
1 & 0 & 0 & 1 \\
0 & 1 & -1 & 0 \\
0 & 1 & 1 & 0 \\
-1 & 0 & 0 & 1
\end{array}\right), \quad B^{-1}=B^{T}=\frac{1}{\sqrt{2}}\left(\begin{array}{cccc}
1 & 0 & 0 & -1 \\
0 & 1 & 1 & 0 \\
0 & -1 & 1 & 0 \\
1 & 0 & 0 & 1
\end{array}\right) .
$$

It has the exponential form,

$$
B=e^{i \frac{\pi}{4}\left(\sigma_{1} \otimes \sigma_{2}\right)}=\cos \frac{\pi}{4}+i \sin \frac{\pi}{4}\left(\sigma_{1} \otimes \sigma_{2}\right)
$$

with the following interesting properties:

$$
B^{2}=i \sigma_{1} \otimes \sigma_{2}, \quad B^{4}=-\mathbb{1}_{4}, \quad B^{8}=\mathbb{1}_{4}, \quad B=\frac{1}{\sqrt{2}}\left(\mathbb{1}_{4}+B^{2}\right) .
$$

The vector $\vec{v}$ including the four product basis $|i j\rangle$ has its transpose,

$$
\vec{v}^{T}=(|00\rangle,|01\rangle,|10\rangle,|11\rangle), \quad v_{i j}=|i j\rangle, i, j=0,1 .
$$

In terms of the $B$ matrix and product basis $v_{i j}$, there are two ways of generating the Bell states, the first type $(I)$ given by

$$
\begin{aligned}
& (I): \quad\left|\phi^{+}\right\rangle=B|11\rangle, \quad\left|\phi^{-}\right\rangle=B|00\rangle, \\
& \left|\psi^{+}\right\rangle=B|01\rangle, \quad\left|\psi^{-}\right\rangle=-B|10\rangle,
\end{aligned}
$$

and the second type $(I I)$ given by

$$
\begin{aligned}
& (I I): \quad\left|\phi^{+}\right\rangle=B^{T}|00\rangle, \quad\left|\phi^{-}\right\rangle=-B^{T}|11\rangle, \\
& \left|\psi^{+}\right\rangle=B^{T}|10\rangle, \quad\left|\psi^{-}\right\rangle=B^{T}|01\rangle,
\end{aligned}
$$

where the Bell operator acts on the basis $|i j\rangle$ in the way

$$
B|i j\rangle=\sum_{k, l=0}^{1}|k l\rangle B_{k l, i j}=\sum_{k, l=0}^{1}|k l\rangle B_{i j, k l}^{T} .
$$


We work on the first type transformation law $(I)$ between the Bell states and product basis and rewrite the teleportation equation (86) into a new formalism,

$$
\begin{aligned}
& \left(\mathbb{1}_{2} \otimes B\right)(|\psi\rangle \otimes|11\rangle)_{C A B} \\
& =\frac{1}{2}\left(B \otimes \mathbb{1}_{2}\right)\left(|00\rangle \otimes \sigma_{3}|\psi\rangle+|01\rangle \otimes \sigma_{1}|\psi\rangle+|10\rangle \otimes i \sigma_{2}|\psi\rangle+|11\rangle \otimes|\psi\rangle\right)_{C A B} \\
& \equiv\left(B \otimes \mathbb{1}_{2}\right)\left(\vec{v}^{T} \otimes \frac{1}{2} \vec{\sigma}_{11}|\psi\rangle\right)_{C A B}
\end{aligned}
$$

in which the vector $\vec{\sigma}_{11}$ is introduced to be a convenient notation and its transpose is given by

$$
\vec{\sigma}_{11}^{T}=\left(\sigma_{3}, \sigma_{1}, i \sigma_{2}, \mathbb{1}_{2}\right) .
$$

The remaining three teleportation equations are derived in the same way due to local unitary transformations among the Bell states (7). For example,

$$
\begin{aligned}
& |\psi\rangle_{C}\left|\phi^{-}\right\rangle_{A B}=\left(\mathbb{1}_{2} \otimes B\right)(|\psi\rangle \otimes|00\rangle)_{C A B} \\
= & |\psi\rangle_{C} \otimes\left(\mathbb{1}_{2} \otimes \sigma_{3}\right)\left|\phi^{+}\right\rangle_{A B}=\left(\mathbb{1}_{2} \otimes \mathbb{1}_{2} \otimes \sigma_{3}\right)|\psi\rangle_{C}\left|\phi^{+}\right\rangle_{A B}, \\
= & \left(B \otimes \mathbb{1}_{2}\right)\left(\vec{v}^{T} \otimes \frac{1}{2} \sigma_{3} \vec{\sigma}_{11}|\psi\rangle\right)_{C A B}
\end{aligned}
$$

where the local unitary transformation $\mathbb{1}_{2} \otimes \mathbb{1}_{2} \otimes \sigma_{3}$ commutes with $B \otimes \mathbb{1}_{2}$. Similarly, we have the other two equations,

$$
\begin{aligned}
& \left(\mathbb{1}_{2} \otimes B\right)(|\psi\rangle \otimes|01\rangle)_{C A B}=\left(B \otimes \mathbb{1}_{2}\right)\left(\vec{v}^{T} \otimes \frac{1}{2} \sigma_{1} \vec{\sigma}_{11}|\psi\rangle\right)_{C A B}, \\
& \left(\mathbb{1}_{2} \otimes B\right)(|\psi\rangle \otimes-|10\rangle)_{C A B}=\left(B \otimes \mathbb{1}_{2}\right)\left(\vec{v}^{T} \otimes-\frac{1}{2} i \sigma_{2} \vec{\sigma}_{11}|\psi\rangle\right)_{C A B},
\end{aligned}
$$

and then collect four of them into an equation,

$$
\begin{aligned}
& \left(\mathbb{1}_{2} \otimes B\right)\left(|\psi\rangle \otimes \vec{v}^{T}\right)_{C A B}=\left(B \otimes \mathbb{1}_{2}\right)\left(\vec{v}^{T} \otimes \frac{1}{2} \vec{\Sigma}|\psi\rangle\right)_{C A B}, \\
& \left(\vec{v}^{T} \otimes \frac{1}{2} \vec{\Sigma}|\psi\rangle\right)_{C A B}=\left(B^{-1} \otimes \mathbb{1}_{2}\right)\left(\mathbb{1}_{2} \otimes B\right)\left(|\psi\rangle \otimes \vec{v}^{T}\right)_{C A B},
\end{aligned}
$$

in terms of the new matrix $\vec{\Sigma}$ given by

$$
\vec{\Sigma}=\left(\sigma_{3}, \sigma_{2}, i \sigma_{1}, \mathbb{1}_{2}\right) \vec{\sigma}_{11} \text {. }
$$

In the following, we sketch the definitions of the braid group and virtual braid group and then verify the Bell matrix $B$ to form the braid representation and also the virtual braid representation, as motivates us to study the problem how to observe the teleportation from the viewpoint of braid. 


\subsection{Bell matrix and braid representation}

The braid representation $b$-matrix is a $d \times d$ matrix acting on $V \otimes V$ where $V$ is an $d$-dimensional vector space. The symbol $b_{i}$ denotes the braid $b$ acting on the tensor product $V_{i} \otimes V_{i+1}$. The classical braid group $B_{n}$ is generated by the braids $b_{1}, b_{2}$, $\cdots, b_{n-1}$ satisfying the braid relation,

$$
\begin{aligned}
b_{i} b_{j} & =b_{j} b_{i}, \quad j \neq i \pm 1, \\
b_{i} b_{i+1} b_{i} & =b_{i+1} b_{i} b_{i+1}, \quad i=1, \cdots, n-2 .
\end{aligned}
$$

The virtual braid group $V B_{n}$ is an extension of the classical braid group $B_{n}$ by the symmetric group $S_{n}$ [24. It has both the braids $b_{i}$ and virtual crossings $v_{i}$ which are defined by the virtual crossing relation,

$$
\begin{aligned}
v_{i}^{2} & =\mathbb{1}, \quad v_{i} v_{i+1} v_{i}=v_{i+1} v_{i} v_{i+1}, \quad i=1, \cdots, n-2, \\
v_{i} v_{j} & =v_{j} v_{i}, \quad j \neq i \pm 1,
\end{aligned}
$$

a presentation of the symmetric group $S_{n}$, and the mixed relations:

$$
\begin{aligned}
b_{i} v_{j} & =v_{j} b_{i}, \quad j \neq i \pm 1, \\
b_{i+1} v_{i} v_{i+1} & =v_{i} v_{i+1} b_{i}, \quad i=1, \cdots, n-2 .
\end{aligned}
$$

Let verify the Bell matrix $B$ satisfying the braid relation. On the right handside of (22), we do the algebraic calculation:

$$
\begin{aligned}
& \left(\mathbb{1}_{2} \otimes B\right)\left(B \otimes \mathbb{1}_{2}\right)\left(\mathbb{1}_{2} \otimes B\right) \\
& =\frac{1}{2 \sqrt{2}}\left(\mathbb{1}_{8}+\mathbb{1}_{2} \otimes i \sigma_{1} \otimes \sigma_{2}\right)\left(\mathbb{1}_{8}+i \sigma_{1} \otimes \sigma_{2} \otimes \mathbb{1}_{2}\right)\left(\mathbb{1}_{8}+\mathbb{1}_{2} \otimes i \sigma_{1} \otimes \sigma_{2}\right) \\
& =\frac{i}{\sqrt{2}}\left(\mathbb{1}_{2} \otimes \sigma_{1} \otimes \sigma_{2}+\sigma_{1} \otimes \sigma_{2} \otimes \mathbb{1}_{2}\right)=\frac{1}{\sqrt{2}}\left(\mathbb{1}_{2} \otimes B^{2}+B^{2} \otimes \mathbb{1}_{2}\right)
\end{aligned}
$$

and on its left handside we have the same result, i.e.,

$$
\left(B \otimes \mathbb{1}_{2}\right)\left(\mathbb{1}_{2} \otimes B\right)\left(B \otimes \mathbb{1}_{2}\right)=\frac{1}{\sqrt{2}}\left(\mathbb{1}_{2} \otimes B^{2}+B^{2} \otimes \mathbb{1}_{2}\right) .
$$

Now we prove the Bell matrix $B$ to satisfy the mixed relation (24) with the permutation matrix $P$ as a virtual crossing,

$$
P=\left(\begin{array}{cccc}
1 & 0 & 0 & 0 \\
0 & 0 & 1 & 0 \\
0 & 1 & 0 & 0 \\
0 & 0 & 0 & 1
\end{array}\right), \quad P|i j\rangle=|j i\rangle, \quad i, j=0,1
$$

On the left handside of (24), we have

$$
\left(\mathbb{1}_{2} \otimes B\right)\left(P \otimes \mathbb{1}_{2}\right)\left(\mathbb{1}_{2} \otimes P\right)(|i\rangle \otimes|j\rangle \otimes|k\rangle)=\left(\mathbb{1}_{2} \otimes B\right)(|k\rangle \otimes|i j\rangle)
$$


and on its right handside, we do the following calculation,

$$
\begin{aligned}
& \left(P \otimes \mathbb{1}_{2}\right)\left(\mathbb{1}_{2} \otimes P\right)\left(B \otimes \mathbb{1}_{2}\right)(|i\rangle \otimes|j\rangle \otimes|k\rangle) \\
& =\sum_{k, l=0}^{1} B_{i^{\prime} j^{\prime}, i j}\left(P \otimes \mathbb{1}_{2}\right)\left(\mathbb{1}_{2} \otimes P\right)\left(\left|i^{\prime} j^{\prime}\right\rangle \otimes|k\rangle\right) \\
& =\sum_{k, l=0}^{1} B_{i^{\prime} j^{\prime}, i j}\left(|k\rangle \otimes\left|i^{\prime} j^{\prime}\right\rangle\right)=\left(\mathbb{1}_{2} \otimes B\right)(|k\rangle \otimes|i j\rangle) .
\end{aligned}
$$

Around the fact that the Bell matrix forms the braid representation, recently, there are interesting research progress: the Bell matrix recognized as a universal quantum gate 10, 31; Yang-Baxterization of the Bell matrix 11, 12; joint paper with Jing and Ge on new types of quantum algebras via the RTT relation 32]; the Markov trace [10] and related extraspecial 2-groups [33. We will propose several kinds of braid interpretations for the teleportation in view of the formulation (20) of the teleportation equation in terms of the braid representation.

\subsection{Teleportation swapping and virtual braid group}

Here we suggest an interpretation for the teleportation in the framework the virtual braid group and then apply it to the teleportation equation (8). A nontrivial unitary braid detecting knots or links can be identified with a universal quantum gate transforming a separate state into an entangled one, see [10, 11, 12. Hence we regard a nontrivial unitary braid as a device yielding an entangled source. On the other hand, we suggest virtual crossings $v_{i}$ responsible for the teleportation based on our observation called the teleportation swapping. There are two natural teleportation swapping operators $(P \otimes I d)(I d \otimes P)$ and $(I d \otimes P)(P \otimes I d)$, satisfying the teleportation swapping equations,

$$
\begin{aligned}
& |k\rangle \otimes|i j\rangle=(P \otimes I d)(I d \otimes P)(|i j\rangle \otimes|k\rangle), \\
& |i j\rangle \otimes|k\rangle=(I d \otimes P)(P \otimes I d)|k\rangle \otimes|i j\rangle,
\end{aligned}
$$

which are shown up in Figure 1, the permutation $P$ represented by a virtual crossing with a small circle at the crossing point. Also, our suggestion is based on the fact that virtual crossings $v_{i}$ form a presentation of symmetric or permutation group $S_{n}$. In our understanding, the braid relation (22) builds a connection between topological entanglements and quantum entanglements, while the virtual crossing relation (24) is a sort of formulation of the teleportation equation (8).

In terms of the Bell matrix $B$, the local unitary transformations (17) among the Bell states have the forms to be used in the following,

$$
\begin{aligned}
& \left|\phi^{-}\right\rangle=B|00\rangle=\left(\mathbb{1}_{2} \otimes \sigma_{3}\right) B|11\rangle=\left(\sigma_{3} \otimes \mathbb{1}_{2}\right) B|11\rangle, \\
& \left|\psi^{+}\right\rangle=B|01\rangle=\left(\mathbb{1}_{2} \otimes \sigma_{1}\right) B|11\rangle=\left(\sigma_{1} \otimes \mathbb{1}_{2}\right) B|11\rangle, \\
& \left|\psi^{-}\right\rangle=-B|10\rangle=\left(\mathbb{1}_{2} \otimes-i \sigma_{2}\right) B|11\rangle=\left(i \sigma_{2} \otimes \mathbb{1}_{2}\right) B|11\rangle .
\end{aligned}
$$



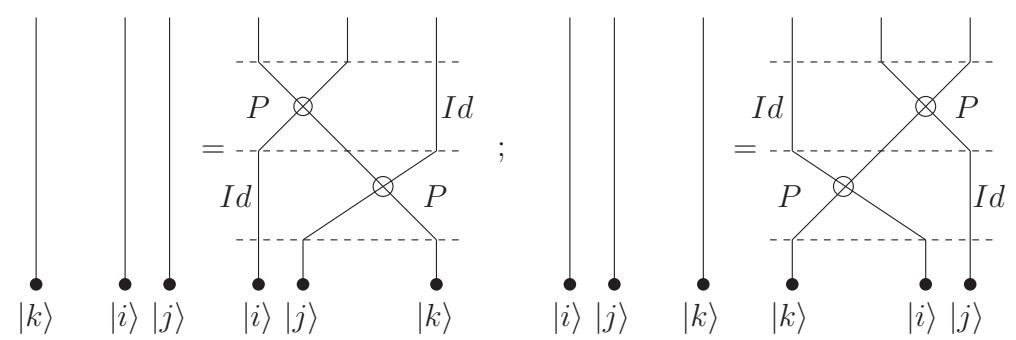

$|k\rangle \otimes|i j\rangle=(P \otimes I d)(I d \otimes P)(|i j\rangle \otimes|k\rangle)$

$|i j\rangle \otimes|k\rangle=(I d \otimes P)(P \otimes I d)(|k\rangle \otimes|i j\rangle)$

Figure 1: Teleportation swapping with permutation $P$ as virtual crossing.

The left handside of the teleportation equation (8) has a formalism in terms of the Bell matrix $B$ and permutation matrix $P$,

$$
\begin{aligned}
& |\psi\rangle_{C} \otimes\left|\phi^{+}\right\rangle_{A B}=\left(\mathbb{1}_{2} \otimes B\right)\left(|\psi\rangle_{C} \otimes|11\rangle_{A B}\right) \\
& =\left(\mathbb{1}_{2} \otimes B\right)\left(P \otimes \mathbb{1}_{2}\right)\left(\mathbb{1}_{2} \otimes P\right)\left(|11\rangle_{C A} \otimes|\psi\rangle_{B}\right),
\end{aligned}
$$

while its right handside leads to

$$
\begin{aligned}
& \frac{1}{2}\left(\left|\phi^{-}\right\rangle_{C A} \sigma_{3}|\psi\rangle_{B}+\left|\psi^{-}\right\rangle_{C A}\left(-i \sigma_{2}\right)|\psi\rangle_{B}+\left|\psi^{+}\right\rangle_{C A} \sigma_{1}|\psi\rangle_{B}+\left|\phi^{+}\right\rangle_{C A}|\psi\rangle_{B}\right) \\
= & \frac{1}{2}\left(\mathbb{1}_{2} \otimes \sigma_{3} \otimes \sigma_{3}+\mathbb{1}_{2} \otimes i \sigma_{2} \otimes i \sigma_{2}+\mathbb{1}_{2} \otimes \sigma_{1} \otimes \sigma_{1}+\mathbb{1}_{8}\right)\left(B \otimes \mathbb{1}_{2}\right)\left(|11\rangle_{C A} \otimes|\psi\rangle_{B}\right) \\
= & \left(\mathbb{1}_{2} \otimes P-\mathbb{1}_{2} \otimes \sigma_{2} \otimes \sigma_{2}\right)\left(B \otimes \mathbb{1}_{2}\right)\left(|11\rangle_{C A} \otimes|\psi\rangle_{B}\right)
\end{aligned}
$$

where the permutation matrix $P$ has been recognized as

$$
P=\frac{1}{2}\left(\mathbb{1}_{4}+\sigma_{1} \otimes \sigma_{1}+\sigma_{2} \otimes \sigma_{2}+\sigma_{3} \otimes \sigma_{3}\right) .
$$

Hence the teleportation equation (8) has a form equivalent to the teleportation swapping,

$$
\begin{aligned}
|\psi\rangle_{C} \otimes\left|\phi^{+}\right\rangle_{A B} & =\left(\mathbb{1}_{2} \otimes P-\mathbb{1}_{2} \otimes \sigma_{2} \otimes \sigma_{2}\right)\left(\left|\phi^{+}\right\rangle_{C A} \otimes|\psi\rangle_{B}\right) \\
& =\left(P \otimes \mathbb{1}_{2}\right)\left(\mathbb{1}_{2} \otimes P\right)\left(\left|\phi^{+}\right\rangle_{C A} \otimes|\psi\rangle_{B}\right)
\end{aligned}
$$

which is also directly related to the virtual crossing relation (24),

$$
\begin{aligned}
& \left(\mathbb{1}_{2} \otimes B\right)\left(P \otimes \mathbb{1}_{2}\right)\left(\mathbb{1}_{2} \otimes P\right)\left(|11\rangle_{C A} \otimes|\psi\rangle_{B}\right) \\
& =\left(\mathbb{1}_{2} \otimes P-\mathbb{1}_{2} \otimes \sigma_{2} \otimes \sigma_{2}\right)\left(B \otimes \mathbb{1}_{2}\right)\left(|11\rangle_{C A} \otimes|\psi\rangle_{B}\right) \\
& =\left(P \otimes \mathbb{1}_{2}\right)\left(\mathbb{1}_{2} \otimes P\right)\left(B \otimes \mathbb{1}_{2}\right)\left(|11\rangle_{C A} \otimes|\psi\rangle_{B}\right) .
\end{aligned}
$$

The remaining three teleportation equations are derived in the way by applying the local unitary transformations to the teleportation equation (35). For example, the 


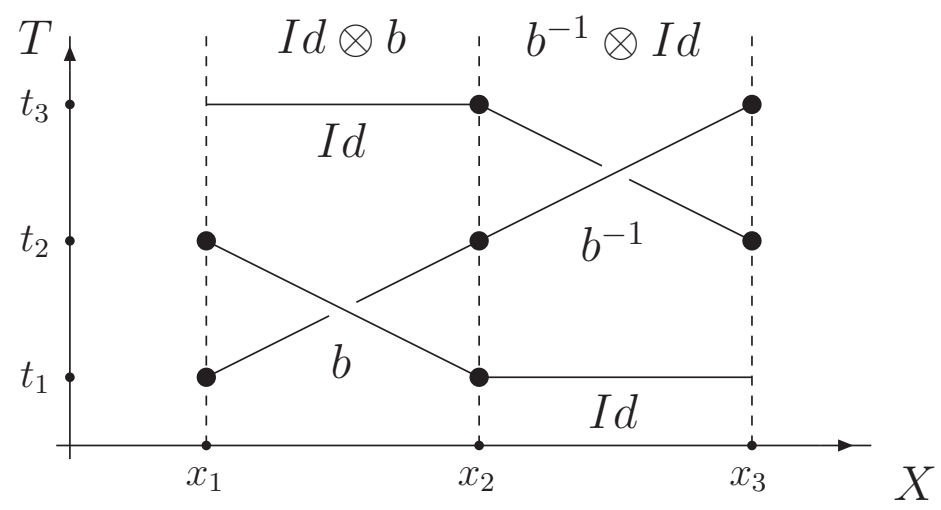

Figure 2: Braid teleportation $\left(b^{-1} \otimes I d\right)(I d \otimes b)$ and crossed measurement.

teleportation equation on the Bell state $\left|\phi^{-}\right\rangle$is obtained to be

$$
\begin{aligned}
& |\psi\rangle_{C} \otimes\left|\phi^{-}\right\rangle_{A B}=\left(\mathbb{1}_{2} \otimes \sigma_{3} \otimes \mathbb{1}_{2}\right)\left(|\psi\rangle_{C} \otimes\left|\phi^{+}\right\rangle_{A B}\right) \\
= & \left(\mathbb{1}_{2} \otimes \sigma_{3} \otimes \mathbb{1}_{2}\right)\left(\mathbb{1}_{2} \otimes P-\mathbb{1} \otimes \sigma_{2} \otimes \sigma_{2}\right)\left(\mathbb{1}_{2} \otimes \sigma_{3} \otimes \mathbb{1}_{2}\right)\left(\left|\phi^{-}\right\rangle_{C A} \otimes|\psi\rangle_{B}\right) \\
= & \left(\mathbb{1}_{2} \otimes P-\mathbb{1}_{2} \otimes \sigma_{1} \otimes \sigma_{1}\right)\left(\left|\phi^{-}\right\rangle_{C A} \otimes|\psi\rangle_{B}\right) .
\end{aligned}
$$

Similarly, the teleportation equations on the Bell states $\left|\psi^{ \pm}\right\rangle$have the same forms as the teleportation swapping,

$$
\begin{aligned}
|\psi\rangle_{C} \otimes\left|\psi^{+}\right\rangle_{A B} & =\left(\mathbb{1}_{2} \otimes P-\mathbb{1}_{2} \otimes \sigma_{3} \otimes \sigma_{3}\right)\left(\left|\psi^{+}\right\rangle_{C A} \otimes|\psi\rangle_{B}\right), \\
|\psi\rangle_{C} \otimes\left|\psi^{-}\right\rangle_{A B} & =\left(\mathbb{1}_{2} \otimes P-\mathbb{1}_{8}\right)\left(\left|\psi^{-}\right\rangle_{C A} \otimes|\psi\rangle_{B}\right),
\end{aligned}
$$

in which the following local unitary transformations of $\left(\mathbb{1}_{2} \otimes P-\mathbb{1}_{2} \otimes \sigma_{2} \otimes \sigma_{2}\right)$ have been exploited,

$$
\begin{aligned}
& \left(\mathbb{1}_{2} \otimes \sigma_{1} \otimes \mathbb{1}_{2}\right)\left(\mathbb{1}_{2} \otimes P-\mathbb{1} \otimes \sigma_{2} \otimes \sigma_{2}\right)\left(\mathbb{1}_{2} \otimes \sigma_{1} \otimes \mathbb{1}_{2}\right)=\mathbb{1}_{2} \otimes P-\mathbb{1}_{2} \otimes \sigma_{3} \otimes \sigma_{3}, \\
& \left(\mathbb{1}_{2} \otimes i \sigma_{2} \otimes \mathbb{1}_{2}\right)\left(\mathbb{1}_{2} \otimes P-\mathbb{1} \otimes \sigma_{2} \otimes \sigma_{2}\right)\left(\mathbb{1}_{2} \otimes i \sigma_{2} \otimes \mathbb{1}_{2}\right)=\mathbb{1}_{2} \otimes P-\mathbb{1}_{8} .
\end{aligned}
$$

As a remark, it is a surprise to realize the virtual braid group to be a kind of natural language describing the teleportation and catch both entanglement and teleportation in the virtual crossing relation (24), as is not explicit in the standard formulation of the teleportation equation (8) .

\subsection{Braid teleportation, crossed measurement and state model}

In view of the formalism (20) of the teleportation equation, we propose the braid teleportation as an extension of the teleportation swapping because the concept of braid is a kind of generalization of permutation. That is to say that the braid configuration like $\left(b^{-1} \otimes I d\right)(I d \otimes b)$ has an ability of realizing the teleportation of a 


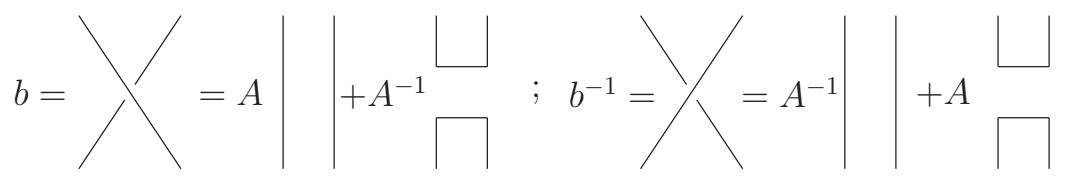

Figure 3: Braid $b$ and its inverse $b^{-1}$ in the state model.

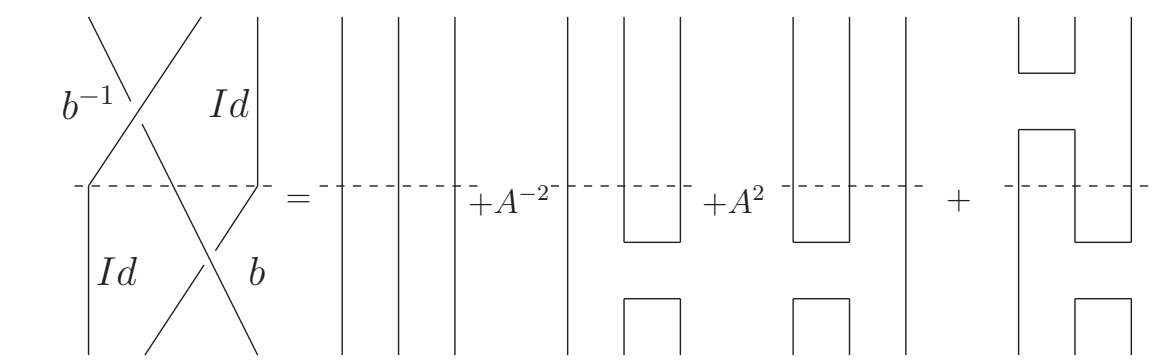

$\left(b^{-1} \otimes I d\right)(I d \otimes b)$

braid teleportation non-teleportation terms

teleportation

Figure 4: Braid teleportation and state model.

quantum state from Charlie to Bob. Here we propose two kinds of interpretations for the braid teleportation. The one is realized by the crossed measurement suggested by Vaidman [26] and the other is related to Kauffman's state model [8] in knot theory.

As the braid is permutation itself, the braid teleportation $\left(b^{-1} \otimes I d\right)(I d \otimes b)$ is the teleportation swapping. As it is not, we explain the braid teleportation from the point of the crossed measurement. We regard a braid or crossing as a device of measurement which is non-local in both space and time. In Figure 2, the two lines of the crossing $b$ represent two observable operations: the first relating the measurement at the space-time point $\left(x_{1}, t_{1}\right)$ to that at the other point $\left(x_{2}, t_{2}\right)$ and the second one relating the measurement at $\left(x_{1}, t_{2}\right)$ to that at $\left(x_{2}, t_{1}\right)$. The crossed measurement $(I d \otimes b)$ plays the role of sending the qubit from Charlie to Alice with a possible rotation. Similarly, the crossed measurement $\left(b^{-1} \otimes I d\right)$ transfers the qubit that Alice obtains from Charlie to Bob also with a possible rotation. This kind of braid teleportation is charming because of the involved crossed measurement and expected relations to the braid statistics of anyons [34] or topological quantum computing.

There is an ambiguity against the braid teleportation $\left(b^{-1} \otimes I d\right)(I d \otimes b)$, which may not have a fixed configuration like $(P \otimes I d)(I d \otimes P)$ from the point of the braid. For example, we rewrite the Bell matrix teleportation $\left(B^{-1} \otimes \mathbb{1}_{2}\right)\left(\mathbb{1}_{2} \otimes B\right)$ into the 
other formalism,

$$
\begin{aligned}
& \left(B^{-1} \otimes \mathbb{1}_{2}\right)\left(\mathbb{1}_{2} \otimes B\right)=\frac{1}{2}\left(\left(\mathbb{1}_{4}-B^{2}\right) \otimes \mathbb{1}_{2}\right)\left(\mathbb{1}_{2} \otimes\left(\mathbb{1}_{4}+B^{2}\right)\right), \\
= & \frac{1}{2}\left(\mathbb{1}_{2} \otimes\left(\mathbb{1}_{4}+B^{2}\right)\right)\left(\left(\mathbb{1}_{4}-B^{2}\right) \otimes \mathbb{1}_{2}\right)+\left(\mathbb{1}_{2} \otimes B^{2}\right)\left(B^{2} \otimes \mathbb{1}_{2}\right) \\
= & \left(\mathbb{1}_{2} \otimes B\right)\left(B^{-1} \otimes \mathbb{1}_{2}\right)+\left(\mathbb{1}_{2} \otimes B^{2}\right)\left(B^{2} \otimes \mathbb{1}_{2}\right)
\end{aligned}
$$

where we make use of the equation,

$$
\left(\mathbb{1}_{2} \otimes B^{2}\right)\left(B^{2} \otimes \mathbb{1}_{2}\right)=-\left(B^{2} \otimes \mathbb{1}_{2}\right)\left(\mathbb{1}_{2} \otimes B\right) .
$$

The braid configuration $\left(\mathbb{1}_{2} \otimes B\right)\left(B^{-1} \otimes \mathbb{1}_{2}\right)$ is different from $\left(B^{-1} \otimes \mathbb{1}_{2}\right)\left(\mathbb{1}_{2} \otimes B\right)$ and also $B^{2}$ is not a braid representation. Therefore we suggest that the braid teleportation $\left(B^{-1} \otimes \mathbb{1}_{2}\right)\left(\mathbb{1}_{2} \otimes B\right)$ has to be understood at a fundamental level. In the following, we go to the state model [8] in knot theory and make it clear why $\left(b^{-1} \otimes I d\right)(I d \otimes b)$ is called the braid teleportation.

The state model 8 disentangles a knot or link in the way presented in Figure 3 where a braid $b$ denoted by a under crossing and its inverse $b^{-1}$ denoted by an over crossing are respectively identified with linear combinations of configurations like two straight lines representing identity and a top cup and a bottom cap representing a projector, and $A, A^{-1}$ are coefficients to be specified by a given state model [8]. Expand the braid teleportation $\left(b^{-1} \otimes I d\right)(I d \otimes b)$ in view of Figure 3 and lead to Figure 4 where the parts above dashed lines are contributed from $\left(b^{-1} \otimes I d\right)$ and the other parts from $(I d \otimes b)$. Observed from Figure 4, it has four diagrammatic terms. The first three can not be responsible for the teleportation but the fourth one is indeed related to the teleportation. This shows that the braid teleportation is made of teleportation and non-teleportation terms and explains the so called ambiguity in the above. In the following, we will study the teleportation in details from the point of the Temperley-Lieb algebra, which is the algebra underlying the state model and leads to the fourth diagrammatic term in Figure 4.

\section{Teleportation and Temperley-Lieb algebra}

In this section, we set up the rules how to draw a diagram for an algebraic expression and apply them to a series of topics around the teleportation. First, we revisit three types of descriptions for the teleportation: the transfer operator, quantum measurement and tight teleportation scheme, in our diagrammatic approach. Second, we propose the Temperley-Lieb algebra or Brauer algebra under the local unitary transformations to underlie the teleportaiton. Third, we study the Temperley-Lieb configurations for the entanglement swapping, quantum computing and multipartite entanglements.

\subsection{Notations for maximally entangled states}

As the maximally entangled states play the key roles in the teleportation, how to make their diagrammatic representations is the bone of our rules. Before devising 
the rules, we fix our notations for them and list their relevant properties.

The vectors $\left|e_{i}\right\rangle, i=0,1, \cdots d-1$ form a set of complete and orthogonal basis for a $d$-dimension Hilbert space $\mathcal{H}$ and the covectors $\left\langle e_{i}\right|$, are chosen for its dual Hilbert space $\mathcal{H}^{*}$,

$$
\sum_{i=0}^{d-1}\left|e_{i}\right\rangle\left\langle e_{i}\right|=\mathbb{1}_{d}, \quad\left\langle e_{j} \mid e_{i}\right\rangle=\delta_{i j}, \quad i, j=1,2, \cdots d-1,
$$

where $\delta_{i j}$ is the Kronecker symbol. The maximally entangled state $|\Omega\rangle$, a quantum state in the two-fold tensor product $\mathcal{H} \otimes \mathcal{H}$ of the Hilbert space $\mathcal{H}$, and its dual state $\langle\Omega|$ have the forms,

$$
|\Omega\rangle=\frac{1}{\sqrt{d}} \sum_{i=0}^{d-1}\left|e_{i} \otimes e_{i}\right\rangle, \quad\langle\Omega|=\frac{1}{\sqrt{d}} \sum_{i=0}^{d-1}\left\langle e_{i} \otimes e_{i}\right|,
$$

The action of a bounded linear operator $M$ in the Hilbert space $\mathcal{H}$ on $|\Omega\rangle$ satisfies

$$
\begin{aligned}
& |\psi\rangle=\left(M \otimes \mathbb{1}_{d}\right)|\Omega\rangle=\frac{1}{\sqrt{d}} \sum_{i=0}^{d-1} M\left|e_{i}\right\rangle \otimes\left|e_{i}\right\rangle \\
& =\frac{1}{\sqrt{d}} \sum_{i, j=0}^{d-1}\left|e_{j}\right\rangle M_{j i} \otimes\left|e_{i}\right\rangle=\frac{1}{\sqrt{d}} \sum_{i, j=0}^{d-1}\left|e_{j}\right\rangle \otimes\left|e_{i}\right\rangle M_{i j}^{T} \\
& =\left(\mathbb{1}_{d} \otimes M^{T}\right)|\Omega\rangle, \quad M_{i j}=\left\langle e_{i}|M| e_{j}\right\rangle, \quad M_{i j}^{T}=M_{j i},
\end{aligned}
$$

which suggests that it is permitted to move the local action of the operator $M$ from the Hilbert space to the other Hilbert space as it acts on $|\Omega\rangle$. The trace of two operators $M^{\dagger}$ and $M^{\prime}$ is represented by an inner product between $|\psi\rangle$ and $\left|\psi^{\prime}\right\rangle$,

$$
\begin{aligned}
\left\langle\psi \mid \psi^{\prime}\right\rangle & \equiv\left\langle\Omega\left|\left(M^{\dagger} \otimes \mathbb{1}_{d}\right)\left(M^{\prime} \otimes \mathbb{1}_{d}\right)\right| \Omega\right\rangle=\frac{1}{d} \sum_{i, j=0}^{d-1}\left\langle e_{i}\left|M^{\dagger} M^{\prime}\right| e_{j}\right\rangle\left\langle e_{i} \mid e_{j}\right\rangle, \\
& =\frac{1}{d} \operatorname{tr}\left(M^{\dagger} M^{\prime}\right), \quad\left|\psi^{\prime}\right\rangle=\left(M^{\prime} \otimes \mathbb{1}_{d}\right)|\Omega\rangle
\end{aligned}
$$

and the inner product with the action of the operator $N_{1} \otimes N_{2}$ has the form in terms of the trace,

$$
\left\langle\psi\left|N_{1} \otimes N_{2}\right| \psi^{\prime}\right\rangle=\frac{1}{d} \operatorname{tr}\left(M^{\dagger} N_{1} M^{\prime} N_{2}^{T}\right) .
$$

The transfer operator $T_{B C}$ sending the quantum state from Charlie to Bob like

$$
T_{B C}|\psi\rangle_{C}=T_{B C} \sum_{k=0}^{d-1} a_{k}\left|e_{k}\right\rangle_{C}=\sum_{k=0}^{d-1} a_{k}\left|e_{k}\right\rangle_{B}=|\psi\rangle_{B}
$$




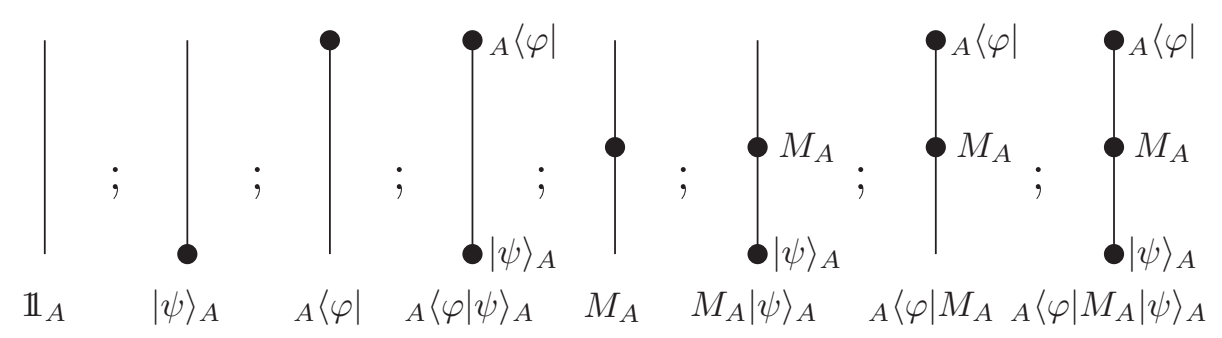

Figure 5: Straight line without or with points.

is recognized to be an inner product in terms of $|\Omega\rangle$,

$$
\begin{aligned}
{ }_{C A}\langle\Omega \mid \Omega\rangle_{A B} & =\frac{1}{d} \sum_{i, j=0}^{d-1}\left({ } _ { C } \left\langlee_{i}\left|\otimes{ }_{A}\left\langle e_{i}\right|\right)\left(\left|e_{j}\right\rangle_{A} \otimes\left|e_{j}\right\rangle_{B}\right)\right.\right. \\
& =\frac{1}{d} T_{B C} \equiv \frac{1}{d} \sum_{i=0}^{d-1}\left|e_{i}\right\rangle_{B} C_{C}\left\langle e_{i}\right|
\end{aligned}
$$

As the above, the maximally entangled state $|\Omega\rangle$ has beautiful properties which will be realized at the level of diagrams. Diagrams catch essential points from the global view so that they are able to express complicated algebraic objects in a clear way. The diagrams from our rules uncover the Temperley-Lieb algebraic structure behind the teleportation and remove mysteries around it from both physical and mathematical sides. The teleportation configuration identifies it with a sort of the flow of quantum information.

\subsection{Rules for drawing diagrams}

Now we present our rules which assign a definite diagram for a given algebraic expression. The diagram consists of straight lines, points, caps and cups. Every element maps to an algebraic term. For example, a cup represents the maximally entangled state $|\Omega\rangle$ and a cap does for its dual state $\langle\Omega|$. The rules have three parts: the first for our convention; the second for straight lines; the third for cups and caps.

Rule 1. Read an algebraic expression such as an inner product from the left to the right and draw a diagram from the top to the bottom.

Rule 2. A straight line of type $A$ without solid points denotes identity of the system $A$, which is a linear combination of projectors, i.e., $\mathbb{1}_{A}=\sum_{i=0}^{d-1}\left|e_{i}\right\rangle_{A}{ }_{A}\left\langle e_{i}\right|$. Straight lines of type $A$ with a top or bottom solid point describe the vector $|\psi\rangle_{A}$, covector ${ }_{A}\langle\varphi|$, and the inner product ${ }_{A}\langle\varphi \mid \psi\rangle_{A}$ for the system $A$, respectively, see Figure 5. Straight lines of type $A$ with a middle or top or bottom solid point describe the operator $M_{A}$, the covector ${ }_{A}\left\langle e_{i}\right| M_{A}$, the vector $M_{A}|\psi\rangle_{A}$ and the inner product ${ }_{A}\left\langle\varphi\left|M_{A}\right| \psi\right\rangle_{A}$, respectively, see Figure 5. A straight line connecting the system $C$ to 


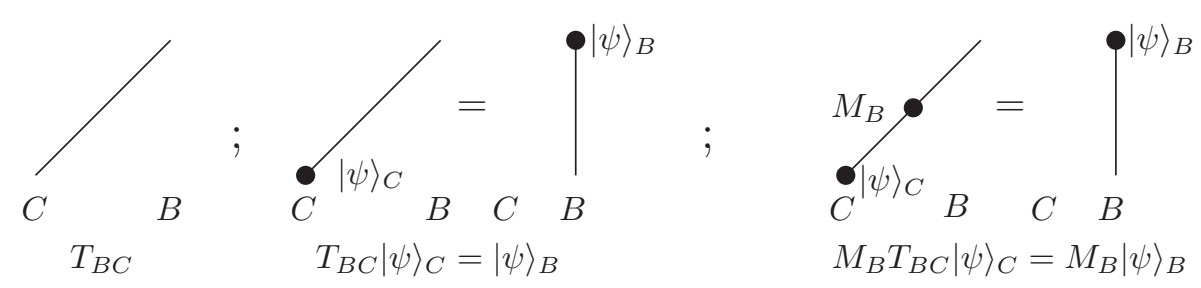

Figure 6: Straight line and transfer operator.

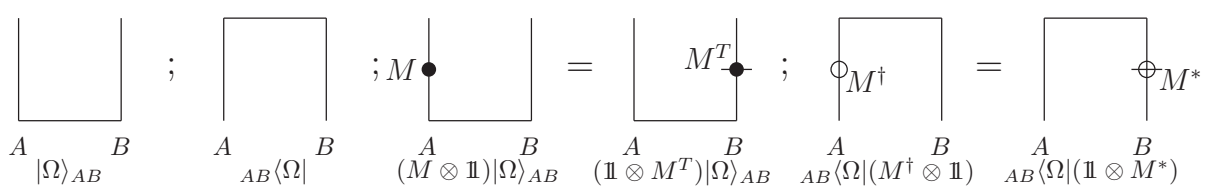

Figure 7: Cup and cap without or with points.

the system $B$ describes the transfer operator $T_{B C}$ and its solid points have the same interpretation as those on the straight line of type $A$, see Figure 6 .

Rule 3. The cup denotes the maximally entangled state $|\Omega\rangle$ and the cap does for its dual $\langle\Omega|$. A cup with a middle solid point in its one branch describes the local action of the operator $M$ on $|\Omega\rangle$ and this solid point can flow to its other branch and becomes a solid point with a cross line representing $M^{T}$ since we already know $\left(M \otimes \mathbb{1}_{d}\right)|\Omega\rangle=\left(\mathbb{1}_{d} \otimes M^{T}\right)|\Omega\rangle$, see Figure 7 . The same things happen for a cap except that the solid point is replaced by a small circle to distinguish the operator $M$ and its transposed and complex version $M^{\dagger}=\left(M^{T}\right)^{*}$, see Figure 7. A cup and a cap have at least three types of combinations for three different cases, see Figure 8. When a cup is at the top and a cap is at the bottom for the same composite system, the diagram is assigned to the projector $|\Omega\rangle\langle\Omega|$. When a cap is at the top and a cup is at the bottom for the same composite system, the diagram describes the inner product $\langle\Omega \mid \Omega\rangle=1$ as a closed circle. Additionally, as a cup has the local action of the operator $M$ and a cap has the local action of the operator $N^{\dagger}$, the resulted circle with a solid point for $M$ and a small circle for $N^{\dagger}$ represent the trace $\frac{1}{d} \operatorname{tr}\left(M N^{\dagger}\right)$. As conventions, we describe a trace of operators by a closed circle with solid points or small circles and assign each cap or cup a normalization factor $\frac{1}{\sqrt{d}}$ and a circle a normalization factor $d$ according to the trace of $\mathbb{1}_{d}$. As a cup is at the bottom for the composite system $\mathcal{H}_{C} \otimes \mathcal{H}_{A}$ and a cap is at the top for the composite system $\mathcal{H}_{A} \otimes \mathcal{H}_{B}$, the diagram is a straight line representing the transfer operator $T_{B C}$ from Charlie to Bob, see Figure 8.

As an example for applying our rules, we draw a diagram for the inner product 


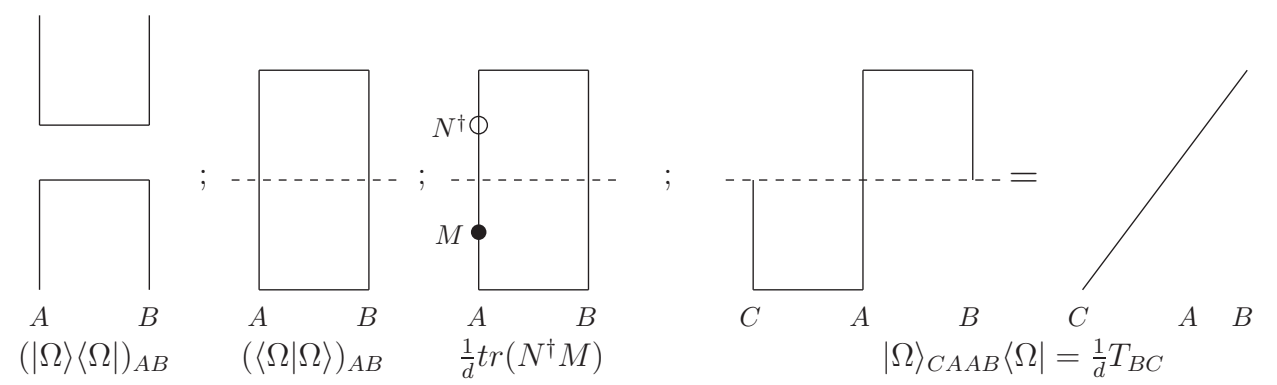

Figure 8: Three kinds of combinations of a cup and a cap.

between $|\phi\rangle=\sum_{i=0}^{d-1} \phi_{i}\left|e_{i}\right\rangle$ and $|\psi\rangle=\sum_{j=0}^{d-1} \psi_{j}\left|e_{j}\right\rangle$ which is calculated in an algebraic way in the following,

$$
\begin{aligned}
& \left\langle\phi \otimes \Omega\left|\left(\mathbb{1}_{d} \otimes M \otimes \mathbb{1}_{d}\right)\right| \Omega \otimes \psi\right\rangle \\
& =\frac{1}{d} \sum_{i, j=0}^{d-1}\left\langle\phi \otimes e_{i} \otimes e_{i}\left|\left(\mathbb{1}_{d} \otimes M \otimes \mathbb{1}_{d}\right)\right| e_{j} \otimes e_{j} \otimes \Omega\right\rangle \\
& =\frac{1}{d} \sum_{i, j=0}^{d-1}\left\langle\phi \mid e_{j}\right\rangle\left\langle e_{i}|M| e_{j}\right\rangle\left\langle e_{i} \mid \psi\right\rangle=\frac{1}{d} \sum_{i, j=0}^{d-1} \phi_{j}^{*} M_{i j} \psi_{i} \\
& =\frac{1}{d} \sum_{i, j=0}^{d-1} \phi_{j}^{*} M_{j i}^{T} \psi_{i}=\frac{1}{d}\left\langle\phi\left|M^{T}\right| \psi\right\rangle,
\end{aligned}
$$

and then derived in our diagrammatic approach, see Figure 9. It consists of three terms: the covector $\langle\phi| \otimes\langle\Omega|$, the local operator $\mathbb{1}_{d} \otimes M \otimes \mathbb{1}_{d}$ and the vector $|\Omega\rangle \otimes|\psi\rangle$. The covector $\langle\psi|$ corresponds to a straight line with a bottom point and the local operator $\mathbb{1}_{d} \otimes M \otimes \mathbb{1}_{d}$ is denoted by a solid point on the cup $|\Omega\rangle$. Move the local operator $M$ from its position to the other branch of the cap, change it to the local operator $M^{T}$ and then allow the bottom cup and the top cap to vanish into a straight line denoting the transfer operator with a normalization factor $\frac{1}{d}$. Therefore it is easy to work with our diagrammatic rules and immediate to obtain the final result.

In the second example, we make a diagrammatic representation for the teleportation equation (8) in Figure 10. The cup denotes the Bell state $\left|\phi^{+}\right\rangle$and the cups with middle solid points $\sigma_{3},-i \sigma_{2}, \sigma_{1}$ denote the Bell states $\left|\phi^{-}\right\rangle,\left|\psi^{-}\right\rangle$and $\left|\psi^{+}\right\rangle$, respectively. The straight line with a bottom solid point denotes the transported state $|\psi\rangle$ and its middle solid points represent the local unitary actions on $|\psi\rangle$.

\subsection{Transfer operator and acausality problem}

Besides its standard description [7] for the teleportation equation (8), the teleportation can be formulated via the transfer operator $T_{B C}$ (47) which sends the quantum 


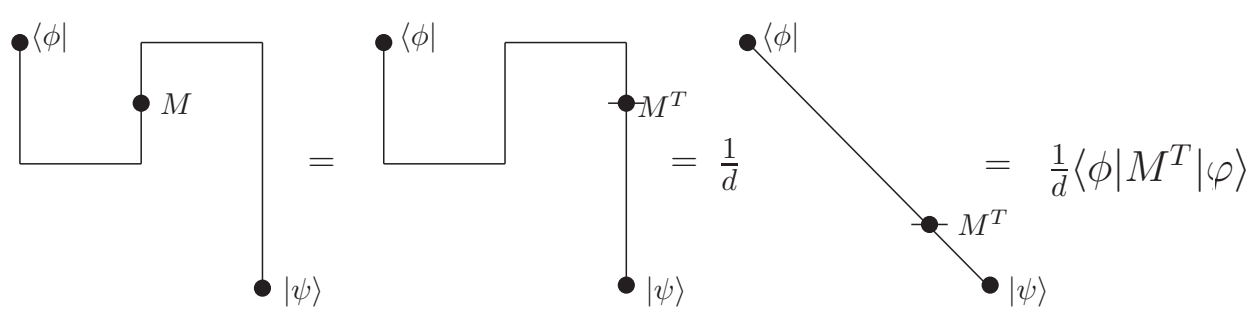

Figure 9: Inner product in terms of a cap and a cup.

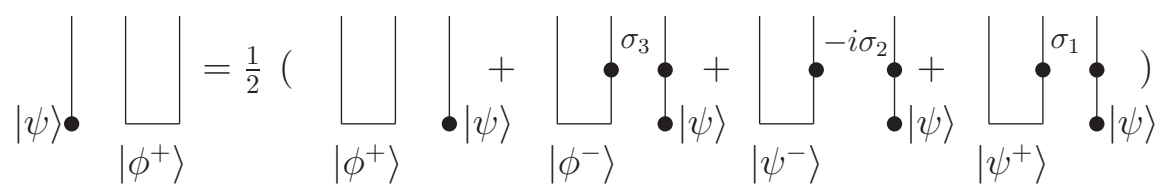

Figure 10: A diagrammatic representation of the teleportation equation (8).

state from Charlie to Bob in the way: $T_{B C}|\psi\rangle_{C}=|\psi\rangle_{B}$. The transfer operator $T_{B C}$ has the form in terms of the maximally entangled state, $T_{B C}={ }_{C A}\langle\Omega \mid \Omega\rangle_{A B}$, and so has a diagrammatic realization according to our rules, see Figure 8 in which the teleportation is regarded as the flow of quantum information.

The entire teleportation process involves local unitary transformations which are not shown up in the formalism of the transfer operator $T_{B C}$ (48). To be general, therefore, we recall the calculation in [27] to represent the transfer operator in terms of the maximally entangled states $|\Phi(U)\rangle_{C A}$ and $\left|\Phi\left(V^{T}\right)\right\rangle_{A B}$ defined by local unitary actions of $U$ and $V^{T}$ on $|\Omega\rangle$, i.e.,

$$
\begin{aligned}
& C A\left\langle\Phi(U) \mid \Phi\left(V^{T}\right)\right\rangle_{A B} \equiv{ }_{C A}\left\langle\Omega\left|\left(U^{\dagger} \otimes \mathbb{1}_{d}\right)\left(V^{T} \otimes \mathbb{1}_{d}\right)\right| \Omega\right\rangle_{A B} \\
& \equiv{ }_{C A B}\left\langle\Omega \otimes \mathbb{1}_{d}\left|\left(U^{\dagger} \otimes \mathbb{1}_{d} \otimes \mathbb{1}_{d}\right)\left(\mathbb{1}_{d} \otimes V^{T} \otimes \mathbb{1}_{d}\right)\right| \mathbb{1}_{d} \otimes \Omega\right\rangle_{C A B} \\
& ={ }_{C A B}\left\langle\Omega \otimes \mathbb{1}_{d}\left|\left(\mathbb{1}_{d} \otimes \mathbb{1}_{d} \otimes V U^{\dagger}\right)\right| \mathbb{1}_{d} \otimes \Omega\right\rangle_{C A B} \\
& =\frac{1}{d} \sum_{i=0}^{d-1} C_{C B}\left\langle e_{i} \otimes \mathbb{1}_{d}\left|\left(\mathbb{1}_{d} \otimes V U^{\dagger}\right)\right| \mathbb{1}_{d} \otimes e_{i}\right\rangle_{C B} \\
& \equiv \frac{1}{d} \sum_{i=0}^{d-1}{ }_{C}\left\langle e_{i}\left|\left(V U^{\dagger}\right)_{B}\right| e_{i}\right\rangle_{B}=\frac{1}{d} \sum_{i=0}^{d-1}\left(V U^{\dagger}\right)_{B}\left|e_{i}\right\rangle_{B}{ }_{C}\left\langle e_{i}\right| \\
& =\frac{1}{d}\left(V U^{\dagger}\right)_{B} T_{B C}
\end{aligned}
$$

which has a special case of $U=V$ given by

$$
\frac{1}{d} T_{B C}={ }_{C A}\left\langle\Phi(U) \mid \Phi\left(U^{T}\right)\right\rangle_{A B} .
$$




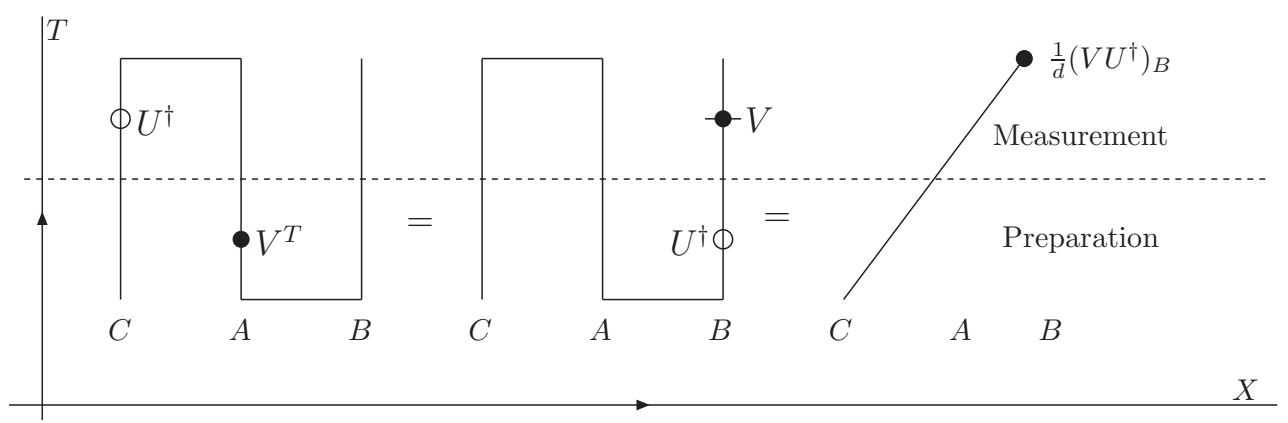

Figure 11: Transfer operator and acausality problem.

In the following, we repeat the above algebraic calculation for the transfer operator $T_{B C}$ at the diagrammatic level and then discuss the acausality problem which becomes explicit after the teleportation is recognized as the flow of quantum information.

From the left to the right, the inner product ${ }_{C A}\left\langle\Phi(U) \mid \Phi\left(V^{T}\right)\right\rangle_{A B}$ has the cap $\langle\Omega|$, identity $\mathbb{1}_{d}$, local unitary operators $U$ and $V^{T}$, identity $\mathbb{1}_{d}$ and cup $|\Omega\rangle$ which are drawn from the top to the bottom, see Figure 11. Move the local operators $U^{\dagger}$ and $V^{T}$ along the lines from their positions to the top point of the system $B$ and obtain the local product $\left(V U^{\dagger}\right)_{B}$ of unitary operators acting on the quantum state that Bob has. The transfer operator $T_{B C}$ has the normalization factor $\frac{1}{d}$ from the vanishing of a cup and a cap. Hence in our diagrammatic approach it is clear that the teleportation can be viewed as a kind of quantum information flow.

But the result $\frac{1}{d}\left(V U^{\dagger}\right)_{B} T_{B C}$ seems to argue that the measurement with the unitary operator $U^{\dagger}$ plays the role before the preparation with the unitary operator $V$. It is not true. Let read Figure 11 in the way where the $T$-axis denotes the time arrow and the $X$-axis denotes the space distance. The quantum information flow starts from the preparation of state, goes to the measurement and come backs to the preparation again and finally goes to the measurement. As a result, it flows from the preparation to the measurement without violating the causality principle.

Note that there are other known approaches to the quantum information flow: the teleportation topology [10, 20] and categorical approach [30. We will compare them with our diagrammatic approach in the next section.

\subsection{Entanglement via measurement (I): teleportation}

Teleportation can be observed from the point of quantum measurement [26, 35]. The only difference from the standard description of the teleportation is that the maximally entangled state $|\Omega\rangle_{A B}$ between Alice and Bob is created in the non-local measurement [26]. Here it is not necessary to go through details involved in the measurement but simply represent it in terms of the projector $(|\Omega\rangle\langle\Omega|)_{A B}$. 


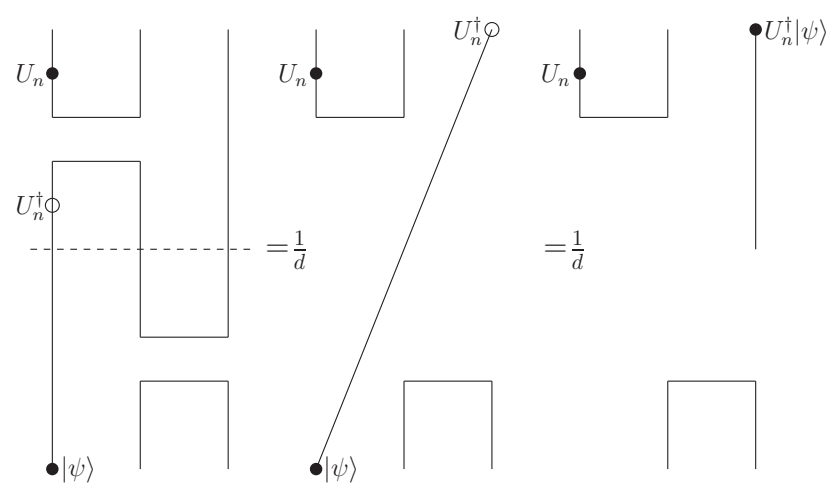

Figure 12: Teleportation based on quantum measurement.

Therefore, the teleportation is determined by two quantum measurements: the one denoted by $(|\Omega\rangle\langle\Omega|)_{A B}$ and the other denoted by $\left(\left|\Omega_{n}\right\rangle\left\langle\Omega_{n}\right|\right)_{C A}$, as leads to a new formulation of the teleportation equation,

$$
\left(\left|\Omega_{n}\right\rangle\left\langle\Omega_{n}\right| \otimes \mathbb{1}_{d}\right)(|\psi\rangle \otimes|\Omega\rangle\langle\Omega|)=\frac{1}{d}\left(\left|\Omega_{n}\right\rangle \otimes \mathbb{1}_{d}\right)\left(\mathbb{1}_{d} \otimes\left(\mathbb{1}_{d} \otimes U_{n}^{\dagger}|\psi\rangle\right)\langle\Omega|\right),
$$

where the maximally entangled state $\left|\Omega_{n}\right\rangle$ is the local unitary transformation of $|\Omega\rangle$, i.e., $\left|\Omega_{n}\right\rangle=\left(U_{n} \otimes \mathbb{1}_{d}\right)|\Omega\rangle$, and the set of local unitary operators $U_{n}$ satisfies the orthogonal relation $\operatorname{tr}\left(U_{n}^{\dagger} U_{m}\right)=d \delta_{n m}, n, m=1, \cdots d^{2}$.

Read the teleportation equation (52) from the left to the right and draw the diagram from the top to the bottom in view of our rules, i.e., Figure 12. There is a natural connection between two formalisms (8) and (52) of the teleportation equation. Choose all unitary matrices $U_{n}$ in the way so that they satisfy

$$
\mathbb{1}_{d}=\sum_{i, j=0}^{d-1}\left|e_{i} \otimes e_{j}\right\rangle\left\langle e_{i} \otimes e_{j}\left|=\sum_{n=1}^{d^{2}}\right| \Omega_{n}\right\rangle\left\langle\Omega_{n}\right|, \quad U_{1}=\mathbb{1}_{d}
$$

and then make a summation of all possible teleportation equations like (52) to derive the version (8) of the teleportation equation in the $d$-dimension Hilbert space,

$$
|\psi\rangle \otimes|\Omega\rangle=\frac{1}{d} \sum_{n=1}^{d^{2}}\left|\Omega_{n}\right\rangle \otimes U_{n}^{\dagger}|\psi\rangle .
$$

In the case of $d=2$, the collections of the unitary operators consist of the unit matrix $\mathbb{1}_{2}$ and Pauli matrices $\sigma_{1}, \sigma_{2}, \sigma_{3}$. The Bell measurements are represented by the projectors in terms of the Bell states $\left|\phi^{ \pm}\right\rangle$and $\left|\psi^{ \pm}\right\rangle$. They lead to the same kind of the teleportation equations as (52),

$$
\left(\left|\phi^{-}\right\rangle\left\langle\phi^{-}\right| \otimes \mathbb{1}_{2}\right)\left(|\psi\rangle \otimes\left|\phi^{+}\right\rangle\left\langle\phi^{+}\right|\right)=\frac{1}{2}\left(\left|\phi^{-}\right\rangle \otimes \mathbb{1}_{2}\right)\left(\mathbb{1}_{2} \otimes\left(\mathbb{1}_{2} \otimes \sigma_{3}|\psi\rangle\right)\left\langle\phi^{+}\right|\right),
$$




$$
\begin{aligned}
& \left(\left|\psi^{+}\right\rangle\left\langle\psi^{+}\right| \otimes \mathbb{1}_{2}\right)\left(|\psi\rangle \otimes\left|\phi^{+}\right\rangle\left\langle\phi^{+}\right|\right)=\frac{1}{2}\left(\left|\psi^{+}\right\rangle \otimes \mathbb{1}_{2}\right)\left(\mathbb{1}_{2} \otimes\left(\mathbb{1}_{2} \otimes \sigma_{1}|\psi\rangle\right)\left\langle\phi^{+}\right|\right), \\
& \left(\left|\psi^{-}\right\rangle\left\langle\psi^{-}\right| \otimes \mathbb{1}_{2}\right)\left(|\psi\rangle \otimes\left|\phi^{+}\right\rangle\left\langle\phi^{+}\right|\right)=\frac{1}{2}\left(\left|\psi^{-}\right\rangle \otimes \mathbb{1}_{2}\right)\left(\mathbb{1}_{2} \otimes\left(\mathbb{1}_{2} \otimes-i \sigma_{2}|\psi\rangle\right)\left\langle\phi^{+}\right|\right), \\
& \left(\left|\phi^{+}\right\rangle\left\langle\phi^{+}\right| \otimes \mathbb{1}_{2}\right)\left(|\psi\rangle \otimes\left|\phi^{+}\right\rangle\left\langle\phi^{+}\right|\right)=\frac{1}{2}\left(\left|\phi^{+}\right\rangle \otimes \mathbb{1}_{2}\right)\left(\mathbb{1}_{2} \otimes\left(\mathbb{1}_{2} \otimes|\psi\rangle\right)\left\langle\phi^{+}\right|\right),
\end{aligned}
$$

which has the summation to be the teleportation equation (88) since the Bell states $\left|\phi^{ \pm}\right\rangle$and $\left|\psi^{ \pm}\right\rangle$are verified to satisfy

$$
\mathbb{1}_{2}=\left|\phi^{+}\right\rangle\left\langle\phi^{+}|+| \phi^{-}\right\rangle\left\langle\phi^{-}|+| \psi^{+}\right\rangle\left\langle\psi^{+}|+| \psi^{-}\right\rangle\left\langle\psi^{-}\right| .
$$

The second example is on the continuous teleportation [26]. The maximally entangled state $|\Omega\rangle$ and teleportated state $|\Psi\rangle$ in the continuous case have the forms,

$$
|\Omega\rangle=\int d x|x, x\rangle, \quad|\Psi\rangle=\int d x \psi(x)|x\rangle,
$$

and the other maximally entangled state $\left|\Omega_{\alpha \beta}\right\rangle$ is formulated by the combined action of the $U(1)$ rotation with the translation $T$ on $|\Omega\rangle$, i.e.,

$$
\left|\Omega_{\alpha \beta}\right\rangle=\left(U_{\beta} \otimes T_{\alpha}\right)|\Omega\rangle \equiv \int d x \exp (i \beta x)|x, x+\alpha\rangle, \quad \alpha, \beta \in \mathbb{R}
$$

which is a common eigenvector of the location operator $\mathbf{X} \otimes \mathbb{1}-\mathbb{1} \otimes \mathbf{X}$ and conjugate momentum operator $\mathbf{P} \otimes \mathbb{1}+\mathbb{1} \otimes \mathbf{P}$,

$$
(\mathbf{X} \otimes \mathbb{1}-\mathbb{1} \otimes \mathbf{X})\left|\Omega_{\alpha \beta}\right\rangle=-\alpha\left|\Omega_{\alpha \beta}\right\rangle, \quad(\mathbf{P} \otimes \mathbb{1}+\mathbb{1} \otimes \mathbf{P})\left|\Omega_{\alpha \beta}\right\rangle=2 \beta\left|\Omega_{\alpha \beta}\right\rangle .
$$

The teleportation equation of the type (52) is obtained to be

$$
\left(\left|\Omega_{\alpha \beta}\right\rangle\left\langle\Omega_{\alpha \beta}\right| \otimes \mathbb{1}\right)(|\Psi\rangle \otimes|\Omega\rangle)=\left(\left|\Omega_{\alpha \beta}\right\rangle \otimes \mathbb{1}\right)\left(\mathbb{1} \otimes \mathbb{1} \otimes U_{-\beta} T_{\alpha}|\Psi\rangle\right)
$$

which has the same diagrammatic representation as Figure 12.

Note that the continuous teleportation is a simple generalization of the discrete teleportation without essential conceptual changes, as is explicit in our diagrammatic approach. Importantly, the diagrammatic representation for the teleportation based on measurement, Figure 12 is a key clue to propose the Temperley-Lieb algebra to be underlying the teleportation because it is a standard configuration in the diagrammatic representation for the Temperley-Lieb algebra.

\subsection{Tight teleportation and dense coding schemes}

The tight teleportation and dense coding schemes [21] require that all involved finite Hilbert spaces are the same $d$ dimensional and the classical channel distinguishes $d^{2}$ signals. All examples we treated in the above belong to the tight class. We build the same notations as in [21]. The density operator $\rho$ is a positive operator with a normalized trace. Charlie has his density operator $\rho_{C}=\left|\phi_{1}\right\rangle\left\langle\phi_{2}\right|$ which is the 
quantum state to be sent to Bob. Alice and Bob share the maximally entangled state $\omega_{A B}=(|\Omega\rangle\langle\Omega|)_{A B}$. The set of observables $\omega_{n}, n=1,2, \cdots d^{2}$ over an output parameter space is a collection of bounded linear operators on the Hilbert space $\mathcal{H}$. Alice makes the Bell measurement in the composite system between Charlie and her, and she chooses her observables $\omega_{n}$ to be local unitary transformations on the maximally entangled state $|\Omega\rangle\langle\Omega|$,

$$
\omega_{n}=\left|\Omega_{n}\right\rangle\left\langle\Omega_{n}|, \quad| \Omega_{n}\right\rangle=\left(U_{n} \otimes \mathbb{1}_{d}\right)|\Omega\rangle, \quad n=1,2, \cdots d^{2}
$$

where the unitary operators $U_{n}$ satisfying the orthogonal condition $\operatorname{tr}\left(U_{n}^{\dagger} U_{m}\right)=$ $d \delta_{n m}$ lead to

$$
\left\langle\Omega_{n} \mid \Omega_{m}\right\rangle=\delta_{n m}, \quad \sum_{n=1}^{d^{2}} \omega_{n}=\mathbb{1}_{d}, \quad n, m=1, \cdots d^{2} .
$$

As Bob gets the message denoted by $n$ from Alice and then applies the transformation $T_{n}$ on his observable $\mathcal{O}$, which are given by

$$
T_{n}(\mathcal{O})=U_{n}^{\dagger} \mathcal{O} U_{n}, \quad \mathcal{O}=\left|\psi_{1}\right\rangle\left\langle\psi_{2}\right|, \quad n=1,2, \cdots d^{2} .
$$

The operator $T_{n}$ defined this way is called a channel, a complete positive linear operator and normalized as $T_{n}\left(\mathbb{1}_{d}\right)=\mathbb{1}_{d}$.

In terms of $\rho_{C}, \omega_{A B},\left(\omega_{n}\right)_{C A}$ and $T_{n}\left(\mathcal{O}_{B}\right)$, the tight teleportation scheme is summarized in the equation

$$
\sum_{n=1}^{d^{2}} \operatorname{tr}\left((\rho \otimes \omega)\left(\omega_{n} \otimes T_{n}(\mathcal{O})\right)\right)=\operatorname{tr}(\rho \mathcal{O}) .
$$

It catches the aim of a successful teleportation, i.e., Charlie makes the measurement in his system as he does in Bob's system although they are far away from each other. It can be proved after some algebraic calculation. The term containing the message $n$ has the form

$$
\begin{aligned}
& \text { term } m_{n}=\operatorname{tr}\left(\left(\left|\phi_{1}\right\rangle\left\langle\phi_{2}|\otimes| \Omega\right\rangle\langle\Omega|\right)\left(\left|\Omega_{n}\right\rangle\left\langle\Omega_{n}|\otimes| U_{n}^{\dagger} \psi_{1}\right\rangle\left\langle\psi_{2}\right| U_{n}\right)\right) \\
& =\left\langle\Omega_{n} \otimes \psi_{2} U_{n} \mid \phi_{1} \otimes \Omega\right\rangle\left\langle\phi_{2} \otimes \Omega \mid \Omega_{n} \otimes U_{n}^{\dagger} \psi_{1}\right\rangle \\
& =\left(\frac{1}{d}\left\langle\psi_{2} \mid \phi_{1}\right\rangle\right)\left(\frac{1}{d}\left\langle\phi_{2} \mid \psi_{1}\right\rangle\right)=\frac{1}{d^{2}} \operatorname{tr}(\rho \mathcal{O})
\end{aligned}
$$

where the inner product (49) has been applied twice. There are $d^{2}$ distinguished messages denoted by $n$, so we prove (64). It is obvious to do calculation at the diagrammatic level, see the left term of Figure 13. The number of classical channel, $n^{2}$ is the number of all possible teleportation diagrams like the left one in Figure 13. Most importantly, it is this diagram which sheds us the insight of proposing the Temperley-Lieb algebra behind the teleportation.

Similarly, all the tight dense coding schemes are concluded in the equation

$$
\operatorname{tr}\left(\omega\left(T_{n} \otimes \mathbb{1}_{d}\right)\left(\omega_{m}\right)\right)=\delta_{n m}
$$




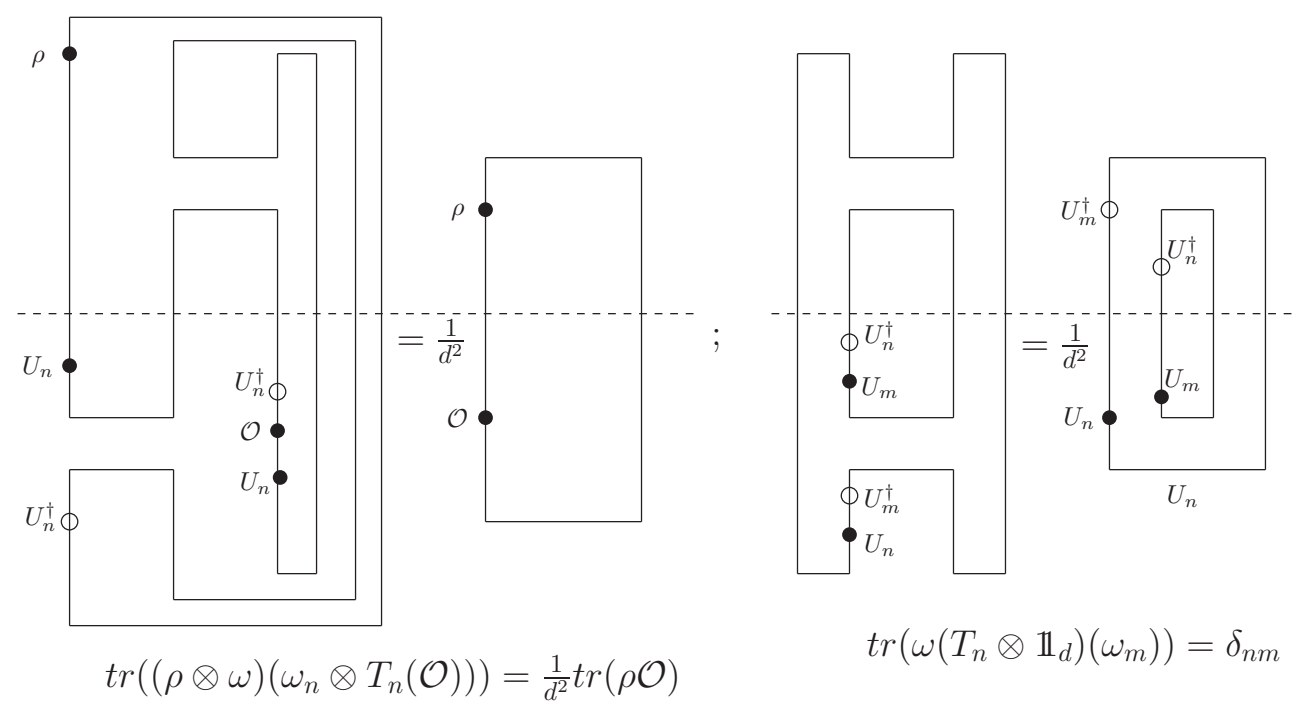

Figure 13: All tight teleportation and dense coding schemes.

which is explained as follows. When Alice and Bob shares the maximally entangled state $|\Omega\rangle_{A B}$, Alice transforms her state by the channel $T_{n}$ to encode the message $n$ and then Bob makes the measurement on observables $\omega_{m}$ of his system. At $n=m$, Bob get the message. The entire process of dense coding is realized in the way,

$$
\begin{aligned}
& \operatorname{tr}\left(|\Omega\rangle\left\langle\Omega\left|\left(U_{n}^{\dagger} \otimes \mathbb{1}_{d}\right)\right| \Omega_{m}\right\rangle\left\langle\Omega_{m}\right|\left(U_{n} \otimes \mathbb{1}_{d}\right)\right) \\
& =\left\langle\Omega\left|U_{n}^{\dagger} \otimes \mathbb{1}_{d}\right| \Omega_{m}\right\rangle\left\langle\Omega_{m}\left|U_{n} \otimes \mathbb{1}_{d}\right| \Omega\right\rangle=\frac{1}{d^{2}}\left(\operatorname{tr}\left(U_{n}^{\dagger} U_{m}\right)\right)^{2}=\delta_{n m}
\end{aligned}
$$

which is the equation (66) and also proved in our diagrammatic approach, see the right term of Figure 13.

Note that the tight teleportation scheme catches all elements of the teleportation and unifies them from the point of the global view into an algebraic or diagrammatic equation. It differs from the other approaches such as the standard description [7, the transfer operator [27] and quantum measurement [26], which all observe the teleportation from the local point of view.

\subsection{Temperley-Lieb algebra and maximally entangled states}

We propose the Temperley-Lieb algebra to underlie the teleportation and make it clear in the following. The Temperley-Lieb algebra $T L_{n}$ is generated by identity $I d$ and $n-1$ hermitian projectors $e_{i}$ satisfying

$$
\begin{aligned}
e_{i}^{2} & =e_{i}, \quad\left(e_{i}\right)^{\dagger}=e_{i}, \quad i=1, \ldots, n-1, \\
e_{i} e_{i \pm 1} e_{i} & =\lambda^{-2} e_{i}, \quad e_{i} e_{j}=e_{j} e_{i}, \quad|i-j|>1,
\end{aligned}
$$




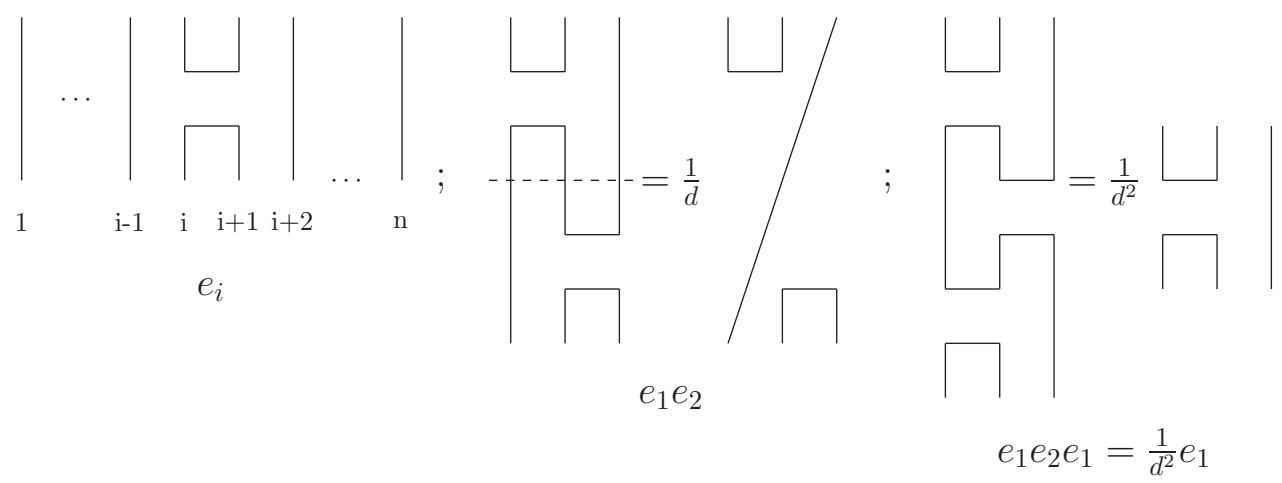

Figure 14: Generator $e_{i}$, multiplication $e_{1} e_{2}$ and axiom for the $T L_{3}(d)$ algebra.

in which $\lambda$ is called loop parameter ${ }^{2}$. Let set up the representation of the $T L_{n}(\lambda)$ algebra in terms of the maximally entangled state $\omega$, a projector,

$$
\omega=|\Omega\rangle\left\langle\Omega\left|=\frac{1}{d} \sum_{i, j=0}^{d-1}\right| i i\right\rangle\langle j j|, \quad \omega^{2}=\omega
$$

by defining idempotents $e_{i}$ in the way

$$
e_{i}=(I d)^{\otimes(i-1)} \otimes \omega \otimes(I d)^{\otimes(n-i-1)}, \quad i=1, \cdots n-1 .
$$

For example, the $T L_{3}(d)$ algebra is generated by two idempotents $e_{1}$ and $e_{2}$,

$$
e_{1}=\omega \otimes I d, \quad e_{2}=I d \otimes \omega,
$$

which are proved to satisfy the axiom $e_{1} e_{2} e_{1}=\frac{1}{d^{2}} e_{1}$ in the way

$$
e_{1} e_{2} e_{1}|\alpha \beta \gamma\rangle=\frac{1}{d} \sum_{l=0}^{d-1} e_{1} e_{2}|l l \gamma\rangle \delta_{\alpha \beta}=\frac{1}{d^{3}} \sum_{n=0}^{d-1}|n n \gamma\rangle \delta_{\alpha \beta}=\frac{1}{d^{2}} e_{1}|\alpha \beta \gamma\rangle
$$

and satisfy the axiom $e_{2} e_{1} e_{2}=\frac{1}{d^{2}} e_{2}$ via similar calculation.

To build an apparent connection between the Temperley-Lieb algebra and teleportation, let make rules for the diagrammatic representation of the Temperley-Lieb algebra which is also called the Brauer diagram [25] or Kauffman diagram [36] in literature. It is a planar $(n, n)$ diagram including a hidden rectangle in the plane with hidden $2 n$ distinct points: $n$ on its top edge and $n$ on its bottom edge which are connected by disjoint strings drawn within in the rectangle. The identity is the diagram with all strings vertical, while $e_{i}$ has its $i$ th and $i+1$ th top (and bottom)

\footnotetext{
${ }^{2}$ The notation $e_{i}$ for the idempotent of the Temperley-Lieb algebra is easily confused with that in the basis vector $\left|e_{i}\right\rangle$. When they appear at the same time, we denote $\left|e_{i}\right\rangle$ by $|i\rangle$.
} 


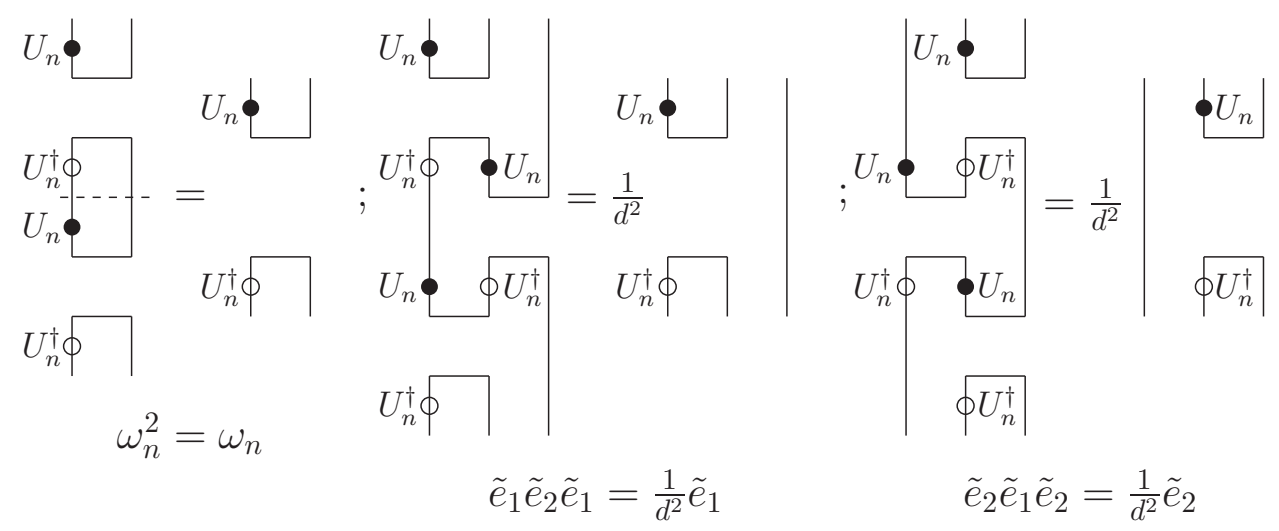

Figure 15: The $T L_{3}(d)$ algebra generated by $\omega_{n}$.

boundary points connected and all other strings vertical. The multiplication $e_{i} e_{j}$ identifies bottom points of $e_{i}$ with corresponding top points of $e_{j}$, removes the common boundary and replaces each obtained loop with a factor $\lambda$. The adjoint of $e_{i}$ is an image under mirror reflection of $e_{i}$ on a horizontal line. In Figure 14, there are diagrammatic representations for $e_{i}, e_{1} e_{2}$ and $e_{1} e_{2} e_{1}=\frac{1}{d^{2}} e_{1}$ with loop parameter $d$.

Therefore, the cup (cap) introduced in our diagrammatic approach is a connected line between top (and bottom) boundary points. Each cup (cap) with a normalization factor $d^{-\frac{1}{2}}$ leaves an additional normalization factor $d^{-\frac{1}{2} N}$ as the number of vanishing cups and caps is $N$, while a closed circle brings a normalization factor $d=\operatorname{tr}\left(\mathbb{1}_{d}\right)$. For examples, in Figure 14, $e_{1} e_{2}$ has a normalization factor $\frac{1}{d}$ from a vanishing cup and a vanishing cap, and $e_{1} e_{2} e_{1}$ has a factor $\frac{1}{d^{2}}$ from four vanishing cups and caps.

In terms of the density matrix $\omega_{n}$ (61) which is the local unitary transformation $U_{n}$ of the maximally entangled state $\omega$, we set up the representation of the Temperley-Lieb algebra, too. For example, the $T L_{3}(d)$ algebra is generated by $\tilde{e}_{1}$ and $\tilde{e}_{2}$,

$$
\tilde{e}_{1}=\omega_{n} \otimes I d, \quad \tilde{e}_{2}=I d \otimes \omega_{n},
$$

which are proved to satisfy the axioms of the Temperley-Lieb algebra in a diagrammatic approach, see Figure 15. Note that the axioms of the Temperley-Lieb algebra are invariant under specified local unitary transformations.

Furthermore, observed from the tight teleportation scheme, the $T L_{3}(d)$ algebra is also built in the way,

$$
e_{1}^{\prime}=\rho \otimes \omega, \quad e_{2}^{\prime}=\omega \otimes \rho, \quad \rho=|\phi\rangle\langle\psi|, \operatorname{tr}(\rho)=1 .
$$

because $\rho$ and $\omega$ and the tensor products between them are all projectors. The axioms of the Temperley-Lieb algebra can be proved in both algebraic and dia- 


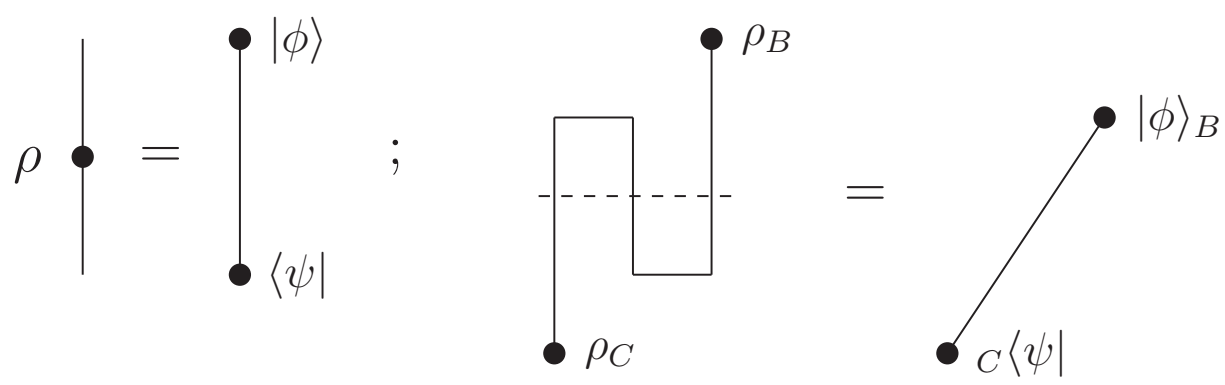

Figure 16: The density state in the transfer operator.

grammatic approaches. Do calculation,

$$
\begin{aligned}
& (\omega \otimes \rho)(\rho \otimes \omega)=\frac{1}{d^{2}} \sum_{i, j=0}^{d-1}((|i i\rangle\langle j j|\otimes| \phi\rangle\langle\psi|)) \sum_{l, m=0}^{d-1}(|\phi\rangle\langle\psi|\otimes| l l\rangle\langle m m|) \\
& =\frac{1}{d^{2}} \sum_{i, j=0}^{d-1} \sum_{l, m=0}^{d-1}\left((|i i \phi\rangle\langle j j \psi|)(|\phi l l\rangle\langle\psi m m|)=\frac{1}{d^{2}} \sum_{i, j=0}^{d-1}|i i \phi\rangle\langle\psi j j|\right.
\end{aligned}
$$

which leads to

$$
\begin{aligned}
& \left.(\omega \otimes \rho)(\rho \otimes \omega)(\omega \otimes \rho)=\frac{1}{d^{3}} \sum_{i, j=0}^{d-1} \sum_{l, m=0}^{d-1}|i i \phi\rangle\langle\psi j j \mid l l \phi\rangle\langle m m \psi|\right) \\
& =\frac{1}{d^{2}} \sum_{i, j=0}^{d-1}|i i \phi\rangle\langle j j \psi|=\frac{1}{d^{2}}(\omega \otimes \rho) .
\end{aligned}
$$

Similarly to prove $e_{2}^{\prime} e_{1}^{\prime} e_{2}^{\prime}=\frac{1}{d^{2}} e_{1}^{\prime}$. The one thing has to be noted, $\rho=|\phi\rangle\langle\psi|$ so that the transfer operator $T_{B C}$ sends a half of $\rho_{C}$, i.e., $|\phi\rangle_{C}$ from Charlie to Bob to form a unit inner product with ${ }_{B}\langle\psi|$, a half of $\rho_{B}$, see Figure 16 .

Moreover, in terms of $\rho$ and $\omega_{n}$, the $T L_{3}(d)$ algebra is set up in the way,

$$
\tilde{e}_{1}^{\prime}=\rho \otimes \omega_{n}, \quad \tilde{e}_{2}^{\prime}=\omega_{n} \otimes \rho, \quad n=1, \cdots d^{2},
$$

if and only if the local unitary transformation $U_{n}$ is a symmetric matrix, as is clear from the diagrammatic point of view, see Figure 15,

$$
U_{n}^{T}=U_{n}, \quad U_{n}^{T} U_{n}^{\dagger}=U_{n}^{*} U_{n}=\mathbb{1}_{d} .
$$

Note that all the Temperley-Lieb diagrams with solid points or small circles created in our diagrammatic approach are found to present simple and clear pictures for algebraic expressions involved in the teleportation. For convenience, we call the Temperley-Lieb category for the Temperley-Lieb algebra under local unitary transformations. 


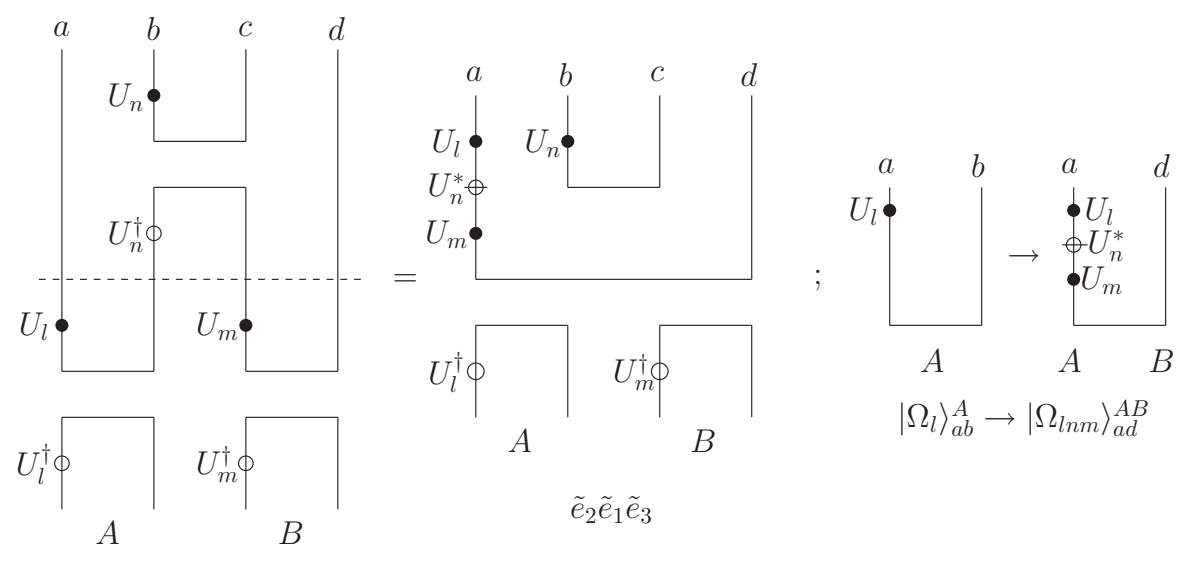

Figure 17: Entanglement swapping and teleportation of entanglement.

\subsection{Entanglement via measurement (II): entanglement swapping}

Entanglement swapping [28] is a sort of experimental technique realizing the entanglement between two independent systems to be a consequence of quantum measurement instead of physical interaction. Let make an example for its theoretical interpretation in terms of projector representing measurement. Alice has a bipartite entangled state $\left|\Omega_{l}\right\rangle_{a b}^{A}$ for particles $a, b$ and Bob has $\left|\Omega_{m}\right\rangle_{c d}^{B}$ for particles $c, d$. They are independently created and do not share common history. Alice applies the measurement denoted by $I d \otimes\left|\Omega_{n}\right\rangle\left\langle\Omega_{n}\right| \otimes I d$ to the product state of $\left|\Omega_{l}\right\rangle_{a b}^{A}$ and $\left|\Omega_{m}\right\rangle_{c d}^{B}$ so that the output called the entanglement swapped state $\left|\Omega_{l n m}\right\rangle_{a d}^{A B}$ is a bipartite entangled state shared by Alice and Bob for particles $a, d$, i.e.,

$$
\begin{aligned}
& \left(I d \otimes\left|\Omega_{n}\right\rangle\left\langle\Omega_{n}\right| \otimes I d\right)\left(\left|\Omega_{l}\right\rangle_{a b}^{A} \otimes\left|\Omega_{m}\right\rangle_{c d}^{B}\right) \\
& =\frac{1}{d}\left(I d \otimes\left|\Omega_{n}\right\rangle \otimes I d\right) \frac{1}{\sqrt{d}} \sum_{i=0}^{d-1}\left(U_{l} U_{n}^{*} U_{m}\left|e_{i}\right\rangle_{a}^{A} \otimes I d \otimes I d \otimes\left|e_{i}\right\rangle_{d}^{B}\right) \\
& \equiv \frac{1}{d}\left(I d \otimes\left|\Omega_{n}\right\rangle \otimes I d\right)\left|\Omega_{l n m}\right\rangle_{a d}^{A B} .
\end{aligned}
$$

In other words, the entanglement swapping reduces a four-particle state $\left|\Omega_{l}\right\rangle_{a b}^{A} \otimes$ $\left|\Omega_{m}\right\rangle_{c d}^{B}$ to a bipartite entangled state $\left|\Omega_{l n m}\right\rangle_{a d}^{A B}$ via the entangling measurement.

Read the entanglement swapping equation (79) from the left to the right and draw a diagram from the top to the bottom according to our diagrammatic rules. It represents a diagrammatic description for an element $\tilde{e}_{2} \tilde{e}_{1} \tilde{e}_{3}$ of the Temeperly-Lieb category, i.e.,

$$
\tilde{e}_{2} \tilde{e}_{1} \tilde{e}_{3}=\left(I d \otimes \omega_{n} \otimes I d\right)\left(\omega_{l} \otimes I d \otimes I d\right)\left(I d \otimes I d \otimes \omega_{m}\right),
$$

which is shown up in the left term of Figure 17. Also, we view the entanglement swapping from the point of the teleportation and hence it changes the entangled 


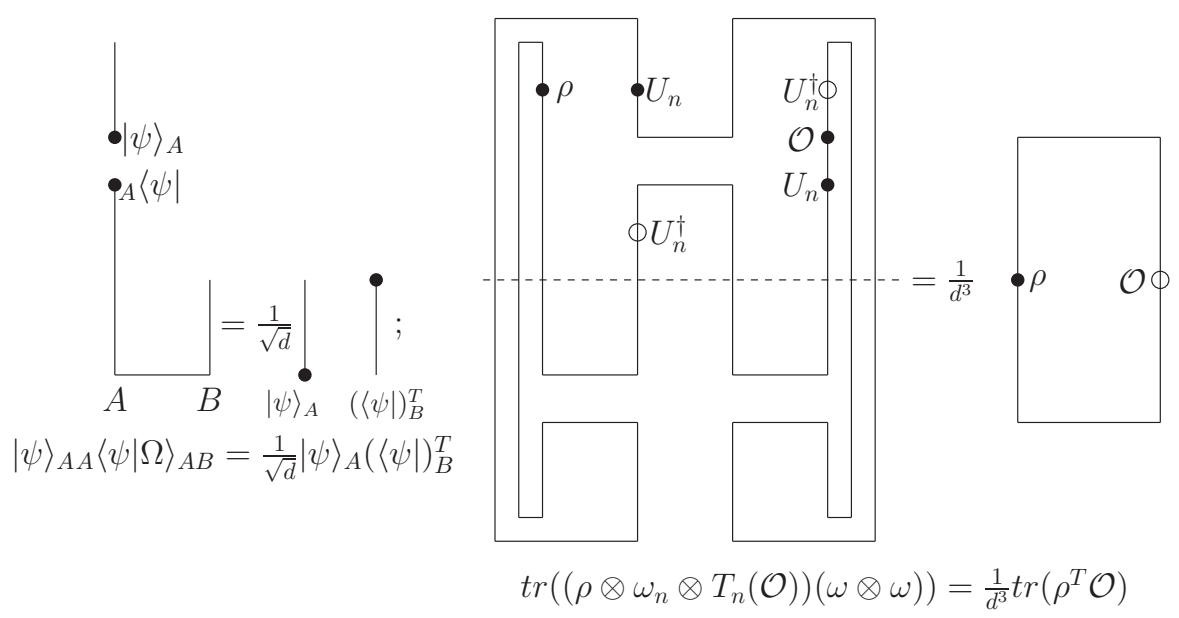

Figure 18: Teleportation via cup and tight entanglement swapping.

state $\left|\Omega_{l}\right\rangle_{a b}^{A}$ shared by particles $a, b$ in Alice's system to the entangled state $\left|\Omega_{l n m}\right\rangle_{a d}^{A B}$ shared by particles $a, d$ in Alice and Bob's composite system.

Furthermore, as the teleportation is denoted by the transfer operator $T_{B C}$, the entanglement swapping can be called the teleportation via the cup state. Alice measures the Bell state $|\Omega\rangle_{A B}$ shared by Bob and her with the projector $|\psi\rangle_{A}{ }_{A}\langle\psi|$ so that she transfers her quantum state to Bob in the way,

$$
\begin{aligned}
& |\psi\rangle_{A}\langle\psi \mid \Omega\rangle_{A B}=\frac{1}{\sqrt{d}}|\psi\rangle_{A} \sum_{i, j=0}^{d-1} A B\left\langle e_{j} \otimes I d\left|\psi_{j}^{*}\right| e_{i} \otimes e_{i}\right\rangle_{A B} \\
& =\frac{1}{\sqrt{d}}|\psi\rangle_{A} \sum_{i=0}^{d-1} \psi_{i}^{*}\left|e_{i}\right\rangle_{B}=\frac{1}{\sqrt{d}}|\psi\rangle_{A}(\langle\psi|)_{B}^{T},
\end{aligned}
$$

which has a diagrammatic representation, see the left term of Figure 18 with $\left(\left\langle e_{i}\right|\right)^{T}=$ $\left|e_{i}\right\rangle$ and is similar to the two-way teleportation performed by the crossed measurement [26, 35].

Moreover, the tight entanglement swapping equation is derived by following the procedure of setting up all tight teleportation and dense coding schemes [21, i.e.,

$$
\sum_{n=1}^{d^{2}} \operatorname{tr}\left(\left(\rho \otimes \omega_{n} \otimes T_{n}(\mathcal{O})\right)(\omega \otimes \omega)\right)=\frac{1}{d} \operatorname{tr}\left(\rho^{T} \mathcal{O}\right)
$$

where the density operator $\rho$ for the particle $a$, observable $\mathcal{O}$ for the particle $d$ and quantum channel $T_{n}(\mathcal{O})$ for the particle $d$ are respectively given by

$$
\rho=\left|\phi_{1}\right\rangle\left\langle\phi_{2}|, \quad \mathcal{O}=| \psi_{1}\right\rangle\left\langle\psi_{2}\right|, \quad T_{n}(\mathcal{O})=U_{n}^{\dagger} \mathcal{O} U_{n}
$$




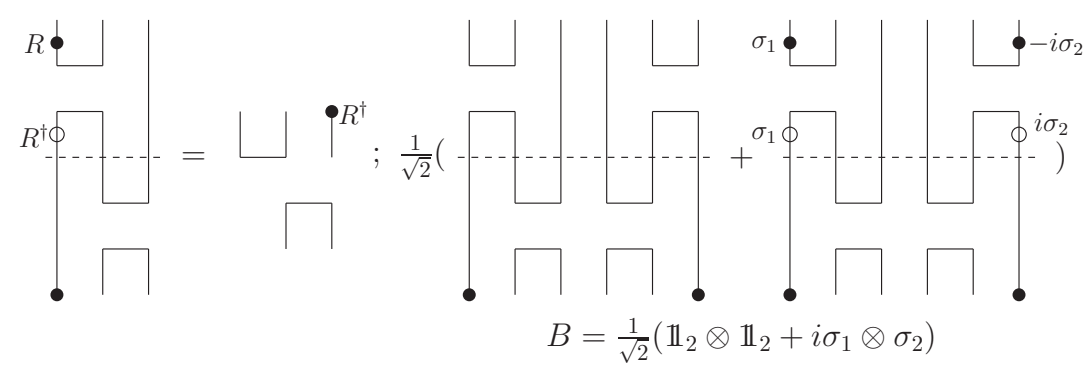

Figure 19: Fault-tolerant gate $R^{\dagger}$ and braid gate $B$ via teleportation.

and the transpose $\rho^{T}$ of the density operator is defined in the way

$$
\rho^{T}=\left(\left|\phi_{1}\right\rangle\left\langle\phi_{2}\right|\right)^{T}=\sum_{i, j=0}^{d-1} \phi_{1 i} \phi_{2 j}^{*}\left(\left|e_{i}\right\rangle\left\langle e_{j}\right|\right)^{T}=\sum_{i, j=0}^{d-1} \phi_{1 i} \phi_{2 j}^{*}\left|e_{j}\right\rangle\left\langle e_{i}\right|, \quad\left(\left\langle e_{i}\right|\right)^{T}=\left|e_{i}\right\rangle .
$$

The tight entanglement swapping equation (82) is proved in our diagrammatic approach in the right term of Figure 18 and also verified in the algebraic way,

$$
\begin{aligned}
& \operatorname{term}_{n}=\operatorname{tr}\left(\left(\left|\phi_{1}\right\rangle\left\langle\phi_{2}|\otimes| \Omega_{n}\right\rangle\left\langle\Omega_{n}\left|\otimes U_{n}^{\dagger}\right| \psi_{1}\right\rangle\left\langle\psi_{2}\right| U_{n}\right)(|\Omega\rangle\langle\Omega|\otimes| \Omega\rangle\langle\Omega|)\right) \\
& =\left\langle\phi_{2} \otimes \Omega_{n} \otimes \psi_{2} U_{n} \mid \Omega \otimes \Omega\right\rangle\left\langle\Omega \otimes \Omega \mid \phi_{1} \otimes \Omega_{n} \otimes U_{n}^{\dagger} \psi_{1}\right\rangle \\
& =\frac{1}{d^{3}}\left(\phi_{2}^{*} \cdot \psi_{2}^{*}\right)\left(\phi_{1} \cdot \psi_{1}\right)
\end{aligned}
$$

which is found to be

$$
\operatorname{tr}\left(\rho^{T} \mathcal{O}\right)=\sum_{i, j=0}^{d-1} \phi_{1 i} \phi_{2 j}^{*}\left\langle e_{i} \mid \psi_{1}\right\rangle\left\langle\psi_{2} \mid e_{j}\right\rangle=\sum_{i, j=0}^{d-1} \phi_{1 i} \phi_{2 j}^{*} \psi_{1 i} \psi_{2 j}^{*}=d^{3} \cdot \text { term }_{n},
$$

as proves (82) since there are $d^{2}$ term $_{n}$ and each term $m_{n}$ is independent of $n$.

Note that the entanglement swapped state $\left|\Omega_{\ln m}\right\rangle_{a d}^{A B}$ plays a special role in the comparison of quantum mechanics with classical physics since it is a quantum state in the quantum world but produced without any direct classical physical interactions.

\subsection{Teleportation and topological quantum computing}

Teleportation has been considered to be a way of performing quantum computation 29]. Under such a proposal, there are both theoretical observations and experimental motivations. Various quantum gates can be easily realized via the teleportation. The teleported state permits the action of local unitary transformations and so the teleportation makes a natural realization of single qubit gates as local unitary transformations. Two-qubit gates are performed in terms of linear combinations of teleportations since they are linear combinations of products of single qubit gates. 


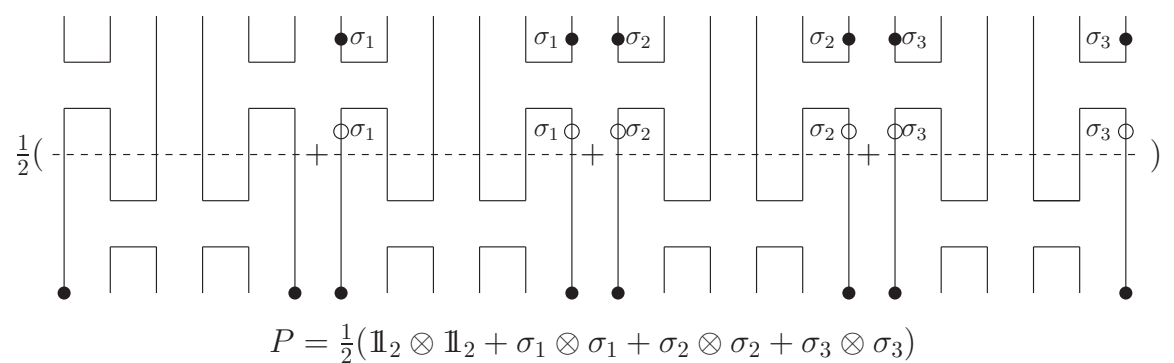

Figure 20: Swap gate in terms of teleportation.

In the experimental sense, single qubit transformations and Bell measurements are basic elements of the teleportation which have been worked out in labs. In contrast with other known approaches to quantum computation, hence, the teleportation relaxes experimental constraints and reduces resources requirements. Additionally, as single qubit transformations are performed fault-tolerantly, we will have the faulttolerant quantum computation [37, 38].

Let make examples for the realizations of quantum gates in terms of the teleportation. A fault-tolerant gate $R$, an element of the Clifford group 37, 38, enters the teleportation via the entangling measurement and then is transported in the form of its conjugation $R^{\dagger}$, see the left term of Figure 19. The Bell matrix $B$ (9) is a typical braid gate and has the form in terms of linear combinations of local unitary transformations,

$$
B=\frac{1}{\sqrt{2}}\left(\mathbb{1}_{2} \otimes \mathbb{1}_{2}+i \sigma_{1} \otimes \sigma_{2}\right)
$$

which is performed in the way shown up in the right term of Figure 19. The Temperley-Lieb category for the braid gate $B$ has two terms and each one consists of two teleportation processes for sending two qubits respectively. Furthermore, the swap gate $P$ has the form given by

$$
P=\frac{1}{2}\left(\mathbb{1}_{2} \otimes \mathbb{1}_{2}+\sigma_{1} \otimes \sigma_{1}+\sigma_{2} \otimes \sigma_{2}+\sigma_{3} \otimes \sigma_{3}\right)
$$

and corresponds to an element of the Temperley-Lieb category in Figure 20, which has four diagrammatic terms and each one represents one term in (88). Similar to the swap gate $P$, the CNOT gate is a linear combination of products of Pauli matrices,

$$
\begin{aligned}
C & =\left(|0\rangle\left\langle 0\left|\otimes \mathbb{1}_{2}+\right| 1\right\rangle\langle 1| \otimes \sigma_{1}\right)=\frac{1}{2}\left(\mathbb{1}_{2}+\sigma_{3}\right) \otimes \mathbb{1}_{2}+\frac{1}{2}\left(\mathbb{1}_{2}-\sigma_{3}\right) \otimes \sigma_{1}, \\
& =\frac{1}{2}\left(\mathbb{1}_{2} \otimes \mathbb{1}_{2}+\mathbb{1}_{2} \otimes \sigma_{1}+\sigma_{3} \otimes \mathbb{1}_{2}-\sigma_{3} \otimes \sigma_{1}\right)
\end{aligned}
$$

which satisfies the basic properties of the CNOT gate,

$$
C|00\rangle=|00\rangle, C|01\rangle=|01\rangle, C|10\rangle=|11\rangle, C|11\rangle=|10\rangle,
$$




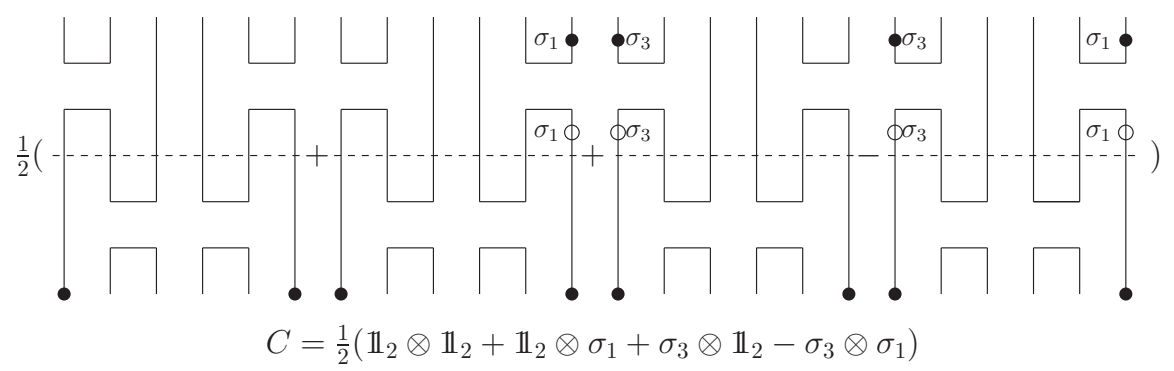

Figure 21: CNOT gate in terms of teleportation.

and hence has a diagrammatic representation in Figure 21.

As the above, the unitary braid gate $B$ and swap gate $P$ have been performed in the context of the teleportation at the conceptual level. This leads to a new approach for topological quantum computing which have two known types of realizations. The one operates with anyons [39] and the other is settled in the Chern-Simons theory [40]. Both involve the unitary braid representation as quantum gates acting on particles like anyons. They are different from our realization of the unitary braid via the teleportation. We do not presume any specific models such as the ChernSimons theory or anyons theory. We build the unitary braid gate just based on its linear combinations of products of local unitary transformations. Hence in our case, topological quantum computing is independent of physical models and so provides a theoretical or conceptual lab for both known approaches.

In terms of a unitary braid gate [10, 11, 12, the knot polynomial can be calculated through quantum simulation of knot on quantum computer, as is different from the way proposed in 41] for computing the Jones polynomial by a devised approximate quantum algorithm. Similarly, with unitary braid gates and swap gate, virtual knots can be simulated via a quantum program. Also, the unitary solutions of the Yang-Baxter equation with spectral parameters [11, 12] can be realized via the Temperley-Lieb category, as leads to the possibility that we study exactly solvable two dimensional quantum field theories or statistical models [13, 14] on quantum computer.

Note that the Temperley-Lieb category, a name for the diagrammatical representation of the Temperley-Lieb algebra under local unitary transformations, is an interesting mathematical subject since it contains abundant objects such as braids, permutation, and so on, and provides a kind of realization of theoretical quantum computer. Also note that a realistic quantum computer requires accurate controllable and fault-tolerantly performed quantum gates and entangling measurements. 

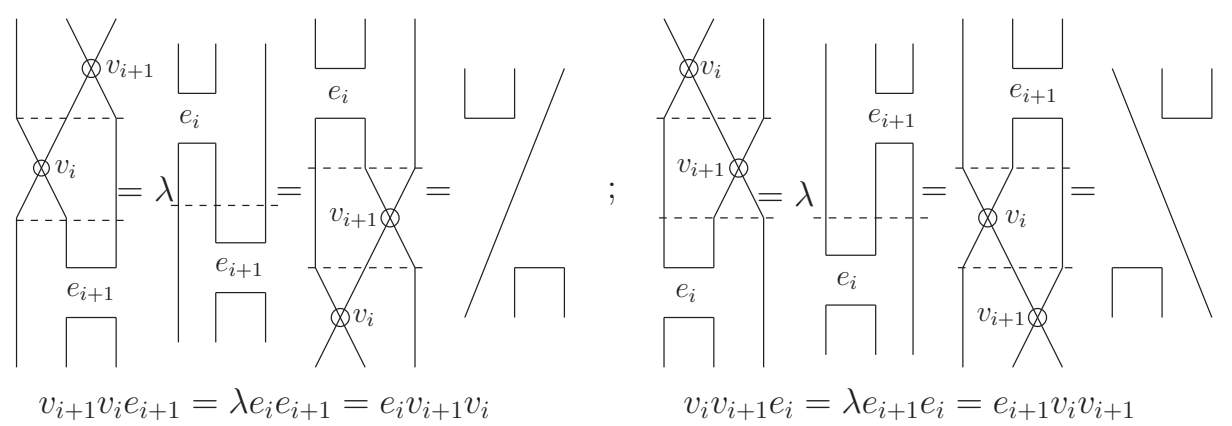

Figure 22: Teleportation, teleportation swapping and Brauer algebra.

\subsection{Teleportation and Brauer algebra}

The teleportation has the same diagrammatic representation as the element $e_{1} e_{2}$ $\left(e_{2} e_{1}\right)$ of the Temperley-Lieb algebra, as has been emphasized and repeated in the above subsections. But there exists a natural question which has to be answered. The teleportation plays the fundamental roles in quantum information theory, but the product $e_{1} e_{2}\left(e_{2} e_{1}\right)$ seems to be only an element of the Temperley-Lieb algebra. Therefore, it is important to to find out in which case the configuration of $e_{1} e_{2}$ $\left(e_{2} e_{1}\right)$ will be crucial in the mathematical sense. Let present the axioms of the Brauer algebra 25] and explain that the teleportation configuration is a bone of this algebra.

The Brauer algebra $D_{n}(\lambda)$ is an extension of the Temperley-Lieb algebra with virtual crossings, $\lambda$ called loop parameter. It has two types of generators: idempotents $e_{i}$ of the Temperley-Lieb algebra $T L_{n}(\lambda)$ satisfying (68) and virtual crossings $v_{i}$ satisfying (23), $i=1, \cdots n-1$. Both generators have to satisfy the mixed relations to determine the Brauer algebra,

$$
\begin{gathered}
(e v / v e): e_{i} v_{i}=v_{i} e_{i}=e_{i}, \quad e_{i} v_{j}=v_{j} e_{i}, \quad j \neq i \pm 1, \\
(v v e): v_{i \pm 1} v_{i} e_{i \pm 1}=\lambda e_{i} e_{i \pm 1}, \quad(e v v): e_{i} v_{i \pm 1} v_{i}=\lambda e_{i} e_{i \pm 1} .
\end{gathered}
$$

For example, the permutation $P$ and maximally entangled state $\omega$ form the Brauer algebra $D_{2}(d)$ with the loop parameter $d$ as $P$ is the virtual crossing and $\omega$ is the idempotent. The mixed relations (91) are obviously verified by substituting

$$
P=\sum_{i, j=0}^{d-1}|i \otimes j\rangle\left\langle j \otimes i\left|, \quad \omega=\frac{1}{d} \sum_{i=0}^{d-1}\right| i \otimes i\right\rangle\langle j \otimes j|
$$

into the axiom $(e v / v e)$,

$$
P \omega=\frac{1}{d} \sum_{i, j=0}^{d-1} \sum_{i^{\prime}, j^{\prime}=0}^{d-1}|i \otimes j\rangle\left\langle j \otimes i \mid i^{\prime} \otimes i^{\prime}\right\rangle\left\langle j^{\prime} \otimes j^{\prime}\right|=\omega=\omega P,
$$


and into the axioms (vve) and (eev),

$$
\begin{aligned}
& \left(\mathbb{1}_{d} \otimes P\right)\left(P \otimes \mathbb{1}_{d}\right)\left(\mathbb{1}_{d} \otimes \omega\right)=d\left(\omega \otimes \mathbb{1}_{d}\right)\left(\mathbb{1}_{d} \otimes \omega\right)=\left(\omega \otimes \mathbb{1}_{d}\right)\left(\mathbb{1}_{d} \otimes P\right)\left(P \otimes \mathbb{1}_{d}\right), \\
& \left(P \otimes \mathbb{1}_{d}\right)\left(\mathbb{1}_{d} \otimes P\right)\left(\omega \otimes \mathbb{1}_{d}\right)=d\left(\mathbb{1}_{d} \otimes \omega\right)\left(\omega \otimes \mathbb{1}_{d}\right)=\left(\mathbb{1}_{d} \otimes \omega\right)\left(P \otimes \mathbb{1}_{d}\right)\left(\mathbb{1}_{d} \otimes P\right),
\end{aligned}
$$

which are proved in a diagrammatic approach, see Figure 22 where $\lambda=d$.

The Figure 22 clearly shows that the teleportation configuration is fundamental for defining the Brauer algebra. It presents an equivalent realization of the teleportation via the swap gate $P$ and Bell measurement, as would be amazing in both the theoretical and experimental sense. It also suggests that the Brauer algebra underlies our previous proposal of the teleportation swapping $(P \otimes I d)(I d \otimes P)$ and virtual braid teleportation. As the braid representation is set up in terms of the Temeperley-Lieb algebra, the virtual braid representation is built with the so called virtual Temperley-Lieb algebra which is the Brauer algebra.

Note that in the joint paper with Kauffman and Werner [22], the virtual TemperleyLieb algebra (the Brauer algebra) will be addressed in details and then applied to topics related to multipartite entanglements.

\subsection{Comments on multipartite entanglements}

This section starts with our diagrammatic rules for terms in an algebraic expression and then applies them to various kinds of examples in order to propose that the Temperley-Lieb algebra (or the Brauer algebra) under local unitary transformations underlies the teleportation. In a general sense, Bell measurements and local unitary transformations are crucial points for the application of our diagrammatic rules, as is explicit in all examples. Here we study additional examples for comments on how to deal multipartite entangled states like the GHZ state or the state $|\chi\rangle$ with our rules. The GHZ state $|G H Z\rangle$ will be rewritten into the form of local unitary transformations on the Bell state,

$$
\begin{aligned}
|G H Z\rangle & =\frac{1}{\sqrt{2}}(|0\rangle \otimes|00\rangle+|1\rangle \otimes|11\rangle) \\
& =\frac{1}{2}(|0\rangle+|1\rangle) \otimes\left|\phi^{+}\right\rangle+\frac{1}{2}(|0\rangle-|1\rangle) \otimes\left|\phi^{-}\right\rangle \\
& =\frac{1}{2}\left(\mathbb{1}_{8}+\sigma_{3} \otimes \mathbb{1}_{2} \otimes \sigma_{3}\right)\left(|\alpha\rangle \otimes\left|\phi^{+}\right\rangle\right)
\end{aligned}
$$

where $|\alpha\rangle=|0\rangle+|1\rangle$ is for conveniences. Similarly, the four-particle state $|\chi\rangle$

corresponds to the form which was used in the construction of the CNOT gate via the teleportation [29],

$$
\begin{aligned}
|\chi\rangle & =\frac{1}{\sqrt{2}}(|00\rangle+|11\rangle)|00\rangle+\frac{1}{\sqrt{2}}(|01\rangle+|10\rangle)|11\rangle \\
& =\frac{1}{\sqrt{2}}\left|\phi^{+}\right\rangle\left(\left|\phi^{+}\right\rangle+\left|\phi^{-}\right\rangle\right)+\frac{1}{\sqrt{2}}\left|\psi^{+}\right\rangle\left(\left|\phi^{+}\right\rangle-\left|\phi^{-}\right\rangle\right)
\end{aligned}
$$




$$
=\frac{1}{\sqrt{2}}\left(\mathbb{1}_{16}+\mathbb{1}_{8} \otimes \sigma_{3}+\mathbb{1}_{2} \otimes \sigma_{1} \otimes \mathbb{1}_{4}-\mathbb{1}_{2} \otimes \sigma_{1} \otimes \mathbb{1}_{2} \otimes \sigma_{3}\right)\left|\phi^{+}\right\rangle\left|\phi^{+}\right\rangle .
$$

As a remark, therefore, we expect that our diagrammatic rules will be applicable to topics such as the Bell inequality, quantum cryptography and so on in which the Bell measurements and local unitary transformations play the fundamental roles.

\section{Comparisons with known approaches}

We propose the Temperley-Lieb algebra under local unitary transformations to be a suitable mathematical framework for the teleportation. To support it, we collect various topics around the teleportation together and deal them with our diagrammatic rules in a systematic way: All involved configurations are elements of the Temperley-Lieb category. To make our proposal more reasonable, we compare it with two known approaches to the quantum information flow: the teleportation topology [10, 20] and strongly compact closed category theory 30].

\subsection{Teleportation topology}

Teleportation topology [10, 20] explains the teleportation as a kind of topological amplitude satisfying the topological condition. There are one to one correspondences between quantum amplitude and topological amplitude. The state preparation (the Dirac ket) describes the creation of two particles from the vacuum and has a diagrammatic representation of the cup state $|C u p\rangle$, while the measurement process (the Dirac bra) denotes the annihilation of two particles and is related to the cap state $\langle C a p|$. The cup state and cap states are associated with the matrices $M$ and $N$ in the way,

$$
|C u p\rangle=\sum_{i, j=0}^{d-1} M_{i j}\left|e_{i} \otimes e_{j}\right\rangle, \quad\langle C a p|=\sum_{i, j=0}^{d-1}\left\langle e_{i} \otimes e_{j}\right| N_{i j}
$$

which have to satisfy the topological condition, i.e., the concatenation of a cup and a cap is a straight line denoted by the identity matrix $N_{i j} M_{j k}=\delta_{i k}$, see Figure 23. However, our diagrammatic teleportation does not have the interpretation of the teleportation topology. The concatenation of a cup and a cap is formulated by the transfer operator which is not identity required by the topological condition. Also, our cup and cap states are the normalized maximally entangled states given by

$$
|C u p\rangle=\frac{1}{\sqrt{d}} \sum_{i=0}^{d-1}\left|e_{i} \otimes e_{i}\right\rangle, \quad\langle\text { Cap }|=\frac{1}{\sqrt{d}} \sum_{i=0}^{d-1}\left\langle e_{i} \otimes e_{i}\right|
$$

which assign a normalization factor $\frac{1}{d}$ to a straight line from the concatenation of a cup and a cap in the teleportation topology. More essentially, our approach underlies the Temperley-Lieb algebra and involves all kinds of combinations of a cup and a 


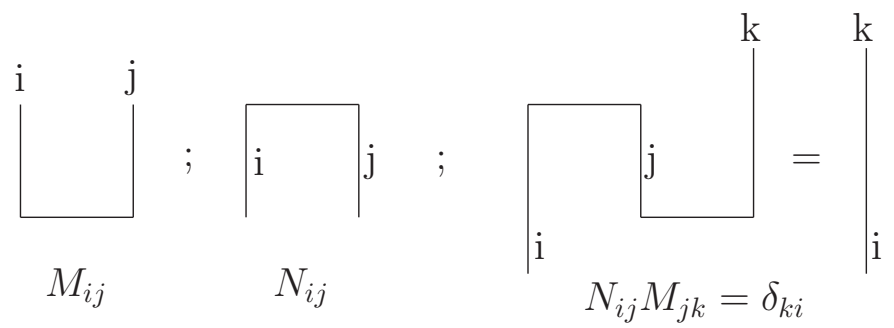

Figure 23: Teleportation topology: cup, cap and topological condition.

cap and especially represents a projector by a cup and a cap which is not considered by the teleportation topology.

Note that the configurations of cups and caps are well known for their applications to the Temperley-Lieb algebra, the braid representation, knot theory and statistics mechanics 8 .

\subsection{Quantum information flow in terms of maps}

The teleportation is a kind of information protocol transporting a quantum state from Charlie to Bob under the help of Alice. To describe it in a unified mathematical formalism, we have to integrate standard quantum mechanics with classical features since the outcomes of measurements are sent to Bob from Alice via classical channels and then Bob carries out a required unitary operation. The one approach has been proposed by Abramsky and Coecke in recent research. They apply the category theory to quantum protocols and suggest the quantum information flow to be described by strongly compact closed categories, see [42, 43] for abstract physical traces; see [30, 44] for quantum information flow; see [45, 46] for the categorical description of quantum protocols; see [47] for diagrammatic quantum mechanics.

To describe the quantum information flow by compositions of a series of maps which are central topics of the category theory, we study an example in details. Set five Hilbert spaces $\mathcal{H}_{i}$ and its dual $\mathcal{H}_{i}^{*}, i=1, \cdots, 5$ and define eight bipartite projectors $P_{\alpha}=\left|\Phi_{\alpha}\right\rangle\left\langle\Phi_{\alpha}\right|, \alpha=1, \cdots, 8$ in which the bipartite vector $\left|\Phi_{\alpha}\right\rangle$ acts on the tensor product $\mathcal{H}_{i} \otimes \mathcal{H}_{i+1}, i=1, \cdots, 4$. In the left diagram of Figure 24, every box represents a bipartite projector $P_{\alpha}$ and the vector $\left|\phi_{C}\right\rangle \in \mathcal{H}_{1}$ that Charlie owns will be transported to Bob who is supposed to obtain the vector $\left|\phi_{B}\right\rangle \in \mathcal{H}_{5}$ through the quantum information flow. The projectors $P_{1}$ and $P_{2}$ picks up the incoming vector in $\mathcal{H}_{2} \otimes \mathcal{H}_{3} \otimes \mathcal{H}_{4} \otimes \mathcal{H}_{5}$ and the projectors $P_{7}$ and $P_{8}$ determine the outgoing vector in $\mathcal{H}_{1} \otimes \mathcal{H}_{2} \otimes \mathcal{H}_{3} \otimes \mathcal{H}_{4}$. The right diagram in Figure 24 presents the quantum information flow from $\left|\phi_{C}\right\rangle$ to $\left|\phi_{B}\right\rangle$ in a clear way. It is drawn according to the permitted and forbidden rules [44]: the flow is forbidden to go through a box from the one side to the other side, and is forbidden to be reflected from the coming position, and has to change its direction from an incoming flow to an outgoing flow 


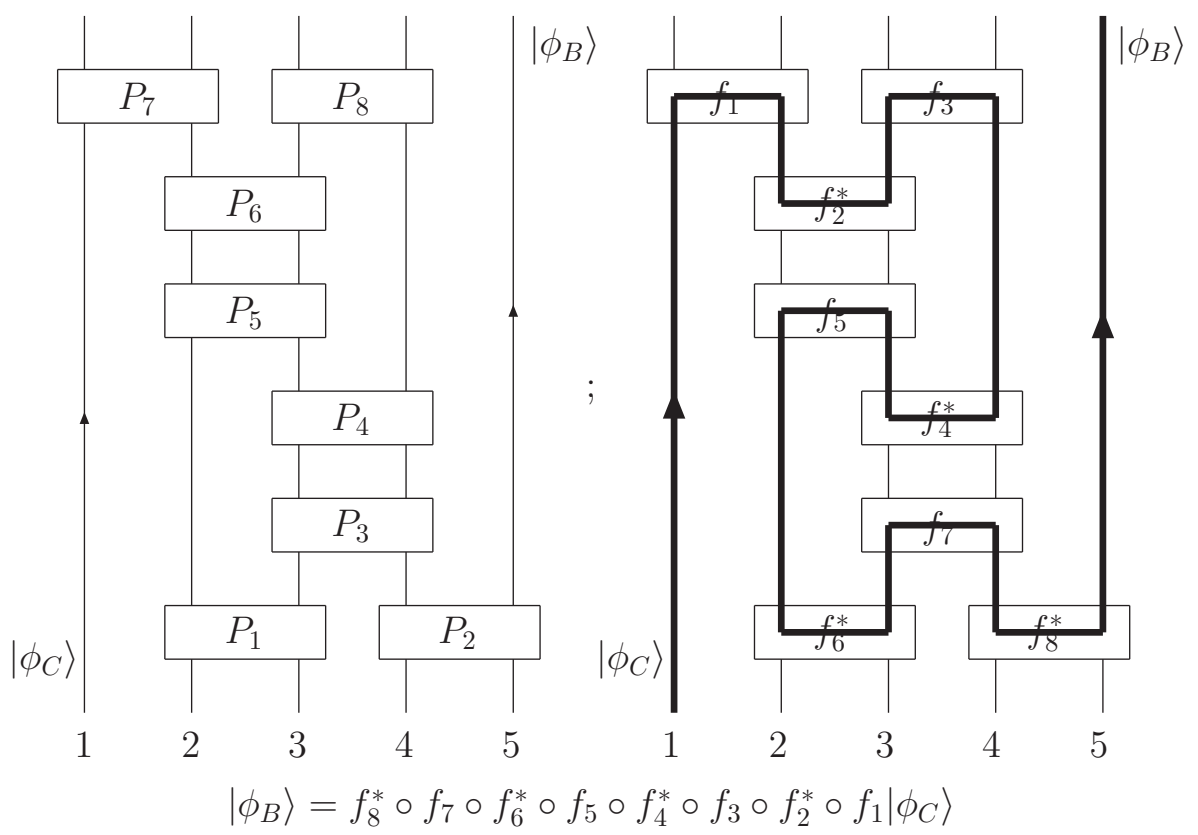

Figure 24: Quantum information flow in the categorical approach.

as it passes through the box. Obviously, there will be many possible pathes from $\left|\phi_{C}\right\rangle$ to $\left|\phi_{B}\right\rangle$ as these rules are not imposed.

Let set up the one to one correspondence between a bipartite vector and a map. There are a $d_{1}$-dimension Hilbert space $\mathcal{H}_{(1)}$ and a $d_{2}$-dimension Hilbert space $\mathcal{H}_{(2)}$. The bipartite vector $|\Phi\rangle$ has the form in terms of the product basis $\left|e_{i}^{(1)}\right\rangle \otimes\left|e_{j}^{(2)}\right\rangle$ of $\mathcal{H}_{(1)} \otimes \mathcal{H}_{(2)}$,

$$
|\Phi\rangle=\sum_{i=0}^{d_{1}-1} \sum_{j=0}^{d_{2}-1} m_{i j}\left|e_{i}^{(1)}\right\rangle \otimes\left|e_{j}^{(2)}\right\rangle, \quad\langle\Phi|=\sum_{i=0}^{d_{1}-1} \sum_{j=0}^{d_{2}-1} m_{i j}^{*}\left\langle e_{i}^{(1)}\right| \otimes\left\langle e_{j}^{(2)}\right|
$$

where $\langle\Phi|$ denotes the dual vector of $|\Phi\rangle$ in the dual product space $\mathcal{H}_{(1)}^{*} \otimes \mathcal{H}_{(2)}^{*}$ with the basis $\left\langle e_{i}^{(1)}\right| \otimes\left\langle e_{j}^{(2)}\right|$. Also, the bipartite vectors $|\Phi\rangle$ or $\langle\Phi|$ are determined by a $d_{1} \times d_{2}$ matrix $M_{d_{1} \times d_{2}}=\left(m_{i j}\right)$. Define two types of maps $f$ and $f^{*}$ in the way,

$$
\begin{aligned}
& f: \mathcal{H}_{1} \rightarrow \mathcal{H}_{2}^{*}, f(\cdot)=\sum_{i=0}^{d_{1}-1} \sum_{j=0}^{d_{2}-1} m_{i j}\left\langle e_{i}^{(1)} \mid \cdot\right\rangle\left\langle e_{j}^{(2)}\right|, \\
& f^{*}: \mathcal{H}_{1}^{*} \rightarrow \mathcal{H}_{2}, \quad f^{*}(\cdot)=\sum_{i=0}^{d_{1}-1} \sum_{j=0}^{d_{2}-1} m_{i j}\left|e_{j}^{(2)}\right\rangle\left\langle\cdot \mid e_{i}^{(1)}\right\rangle
\end{aligned}
$$


and once the product basis are fixed, we have the following bijective correspondences,

$$
|\Phi\rangle \approx\langle\Phi| \approx M \approx f \approx f^{*}
$$

which suggests that we can label the bipartite project box in Figure 24 with the maps $f$ and $f^{*}$ or corresponding matrices.

Let work out the formalism of the quantum information flow in the categorical approach. Consider the projector $P_{7}=\left|\Phi_{7}\right\rangle\left\langle\Phi_{7}\right|$ and introduce the map $f_{1}$ to represent $\left\langle\Phi_{7}\right|$, the half of $P_{7}$,

$$
f_{1}: \mathcal{H}_{1} \rightarrow \mathcal{H}_{2}^{*}, \quad f_{1}\left(\phi_{C}\right)=\left\langle\Phi_{7} \mid \phi_{C}\right\rangle
$$

Similarly, the remaining seven boxes are labeled by the maps $f_{2}^{*}, f_{3}, f_{4}^{*}, f_{5}, f_{6}^{*}, f_{7}$ and $f_{8}^{*}$ which are defined by

$$
\begin{aligned}
& f_{2}^{*}: \mathcal{H}_{2}^{*} \rightarrow \mathcal{H}_{3}, \quad f_{2}^{*} \circ f_{1}\left(\phi_{C}\right)=\left\langle\Phi_{7} \mid \phi_{C} \otimes \Phi_{6}\right\rangle, \\
& f_{3}: \mathcal{H}_{3} \rightarrow \mathcal{H}_{4}^{*}, \quad f_{3} \circ f_{2}^{*} \circ f_{1}\left(\phi_{C}\right)=\left\langle\Phi_{7} \otimes \Phi_{8} \mid \phi_{C} \otimes \Phi_{6}\right\rangle, \\
& f_{4}^{*}: \mathcal{H}_{4}^{*} \rightarrow \mathcal{H}_{3}, \quad f_{4}^{*} \circ f_{3} \circ f_{2}^{*} \circ f_{1}\left(\phi_{C}\right)=\left\langle\Phi_{7} \otimes \Phi_{8} \mid \phi_{C} \otimes \Phi_{6} \otimes \Phi_{4}\right\rangle, \\
& f_{5}: \mathcal{H}_{3} \rightarrow \mathcal{H}_{2}^{*}, \quad f_{5} \circ f_{4}^{*} \circ f_{3} \circ f_{2}^{*} \circ f_{1}\left(\phi_{C}\right)=\left\langle\Phi_{5} \otimes \Phi_{7} \otimes \Phi_{8} \mid \phi_{C} \otimes \Phi_{6} \otimes \Phi_{4}\right\rangle, \\
& f_{6}^{*}: \mathcal{H}_{2}^{*} \rightarrow \mathcal{H}_{3}, \quad f_{7}: \mathcal{H}_{3} \rightarrow \mathcal{H}_{4}^{*}, \quad f_{8}^{*}: \mathcal{H}_{4}^{*} \rightarrow \mathcal{H}_{5}, \\
& f_{6}^{*} \circ f_{5} \circ f_{4}^{*} \circ f_{3} \circ f_{2}^{*} \circ f_{1}\left(\phi_{C}\right)=\left\langle\Phi_{5} \otimes \Phi_{7} \otimes \Phi_{8} \mid \phi_{C} \otimes \Phi_{6} \otimes \Phi_{4} \otimes \Phi_{1}\right\rangle, \\
& f_{7} \circ f_{6}^{*} \circ f_{5} \circ f_{4}^{*} \circ f_{3} \circ f_{2}^{*} \circ f_{1}\left(\phi_{C}\right)=\left\langle\Phi_{3} \otimes \Phi_{5} \otimes \Phi_{7} \otimes \Phi_{8} \mid \phi_{C} \otimes \Phi_{6} \otimes \Phi_{4} \otimes \Phi_{1}\right\rangle,
\end{aligned}
$$

and so the quantum information flow is included in the the form,

$$
\begin{aligned}
& f_{8}^{*} \circ f_{7} \circ f_{6}^{*} \circ f_{5} \circ f_{4}^{*} \circ f_{3} \circ f_{2}^{*} \circ f_{1}\left(\phi_{C}\right) \\
& =\left\langle\Phi_{3} \otimes \Phi_{5} \otimes \Phi_{7} \otimes \Phi_{8} \mid \phi_{C} \otimes \Phi_{6} \otimes \Phi_{4} \otimes \Phi_{1} \otimes \Phi_{2}\right\rangle,
\end{aligned}
$$

namely, it is given by the composition of a series of maps,

$$
\left|\phi_{B}\right\rangle=f_{8}^{*} \circ f_{7} \circ f_{6}^{*} \circ f_{5} \circ f_{4}^{*} \circ f_{3} \circ f_{2}^{*} \circ f_{1}\left|\phi_{C}\right\rangle
$$

where we identify the tensor product $|\Phi\rangle \otimes \mathbb{1}_{d} \otimes \cdots \otimes \mathbb{1}_{d}$ with $|\Phi\rangle$. Additionally, following the rules of the teleportation topology [10, 20] and assigning the matrices $M, N$ to a cup and a cap respectively, we have the quantum information flow in the matrix teleportation,

$$
\left|\phi_{B}\right\rangle=M_{8} \cdot N_{7} \cdot M_{6} \cdot N_{5} \cdot M_{4} \cdot M_{3} \cdot N_{2} \cdot M_{1}\left|\phi_{C}\right\rangle .
$$

Now it is time to make differences clear in a straight way between our diagrammatic teleportation and quantum information flow in the categorical approach. These essential differences are conceptual: both physical and mathematical. On the mathematical side, we think the braid group and Temperley-Lieb algebra behind the teleportation instead of various of maps in the category theory because we are 


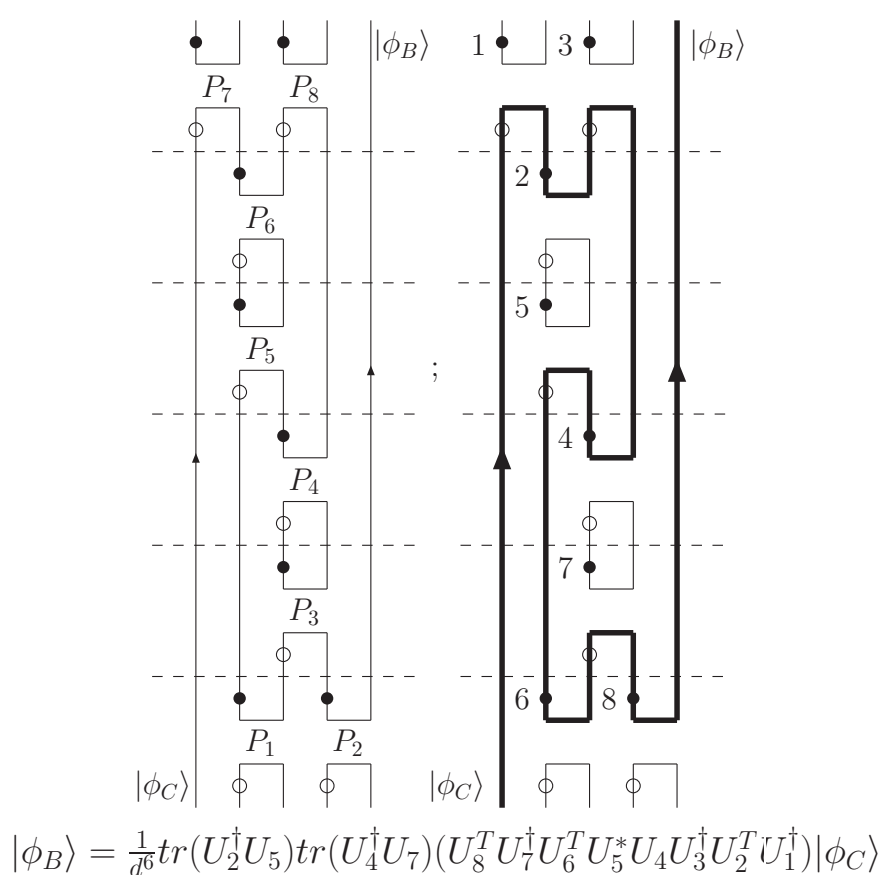

Figure 25: Quantum information flow in the Temperley-Lieb category.

looking for a real and stable bridge between knot theory and quantum information 22. In our approach, the bipartite projector is regarded as an idempotent of the Temperley-Lieb algebra but in the categorical approach only its half, a bipartite vector, has been seriously exploited. On the physical side, we choose the tight teleportation scheme 21] as our definition for the teleportation, i.e., we stick to concepts like the Hilbert space, state, vector and local unitary transformation which are basic ingredients of standard quantum mechanics described by the von Neummann's axioms. But the categorical approach aims at setting up a high-level approach beyond the von Neummann's axioms to describe quantum information theory in a unified mathematical framework.

To explain these differences in details, we revisit the example in Figure 25 and redraw the diagram according to our diagrammatical rules. In Figure 26, every projector consists of a cup and a cap representing the maximally entangled states $|\Omega\rangle$ and $\langle\Omega|$. The solid points $1, \cdots 8$ on the left branch of a cup denote the local unitary transformations $U_{1}, \cdots, U_{8}$ and small circles on the left branch of a cap denote their adjoint operators $U_{1}^{\dagger}, \cdots, U_{8}^{\dagger}$, respectively. Following our rules, the quantum information flow from $\left|\phi_{C}\right\rangle$ to $\left|\phi_{B}\right\rangle$ is determined by the transfer operator,

$$
\left|\phi_{B}\right\rangle=\frac{1}{d^{6}} \operatorname{tr}\left(U_{2}^{\dagger} U_{5}\right) \operatorname{tr}\left(U_{4}^{\dagger} U_{7}\right)\left(U_{8}^{T} \circ U_{7}^{\dagger} \circ U_{6}^{T} \circ U_{5}^{*} \circ U_{4} \circ U_{3}^{\dagger} \circ U_{2}^{\dagger} \circ U_{1}^{\dagger}\right)\left|\phi_{C}\right\rangle
$$

where the normalization factor $\frac{1}{d^{6}}$ is contributed from six vanishing cups and six 
vanishing caps and two traces from two closed circles.

There are at least three remarkable things to be mentioned as Figure 25 is compared with Figure 24. They are related to three questions respectively: why do we denote the projector by itself instead of its half? How to deal with the acausality problem? which types of bijective correspondences between a bipartite vector and a map or matrix would be chosen? In the categorical approach, only the half of a projector is considered in order to make use of the bijective correspondences between a bipartite vector and a map. But obviously observed from our diagrammatic description in Figure 25, our approach not only derives the quantum information flow from $\left|\phi_{C}\right\rangle$ to $\left|\phi_{B}\right\rangle$ in a clear way but also yields a normalization factor from the closed circles. Importantly, the normalization factor contributed by vanishing cups and caps is crucial for the quantum formation flow. For examples, setting eight local unitary operators $U_{i}$ to be identity leads to $\left|\phi_{B}\right\rangle=\frac{1}{d^{4}}\left|\phi_{C}\right\rangle$ and assuming $U_{2}$ and $U_{5}$ (or $U_{4}$ and $U_{7}$ ) orthogonal to each other causes a zero vector to be sent to Bob, $\left|\phi_{B}\right\rangle=0$, no flow! Also, we want to explain the teleportation from the point of quantum measurement since a projector represents a process of measurement in standard quantum mechanics.

The acausality problem becomes explicit in the known approaches to the quantum information flow. But the quantum information flow is only one part of the entire diagram in our diagrammatical approach. In other words, it is not reasonable to argue such questions on the teleportation without considering the whole process from the global view. Hence we choose to represent the projector as the combination

of a cup and a cap instead of a cap or a cup. Furthermore, we apply the bijective correspondence between a local unitary transformation and a bipartite vector or projector, as is different from the choice preferred by the categorical approach. For example, we have

$$
|\psi(U)\rangle=\left(U \otimes \mathbb{1}_{d}\right)|\Omega\rangle, \quad|\psi(U)\rangle \approx U \approx|\psi(U)\rangle\langle\psi(U)|
$$

and so the Bell states (7) are represented by identity or the Pauli matrices

$$
\left|\phi^{+}\right\rangle \approx \mathbb{1}_{2},\left|\phi^{-}\right\rangle \approx \sigma_{3},\left|\psi^{+}\right\rangle \approx \sigma_{1},\left|\psi^{-}\right\rangle \approx i \sigma_{2} .
$$

If we label a projector by a local unitary transformation, then we call the equation (107) the quantum information flow in terms of local unitary transformations.

Note that in the categorical approach the quantum information flow is created in view of additional permitted and forbidden rules [44] but in the Temperley-Lieb category it is derived in a natural way without imposed rules, as is clearly observed by comparing Figure 24 with Figure 25.

\subsection{Teleportation in the strongly compact closed category}

The quantum information flow in terms of compositions of maps naturally leads to its description in the category theory. Here we aim at showing one-to-one correspondences between the quantum information flow and strongly compact category 

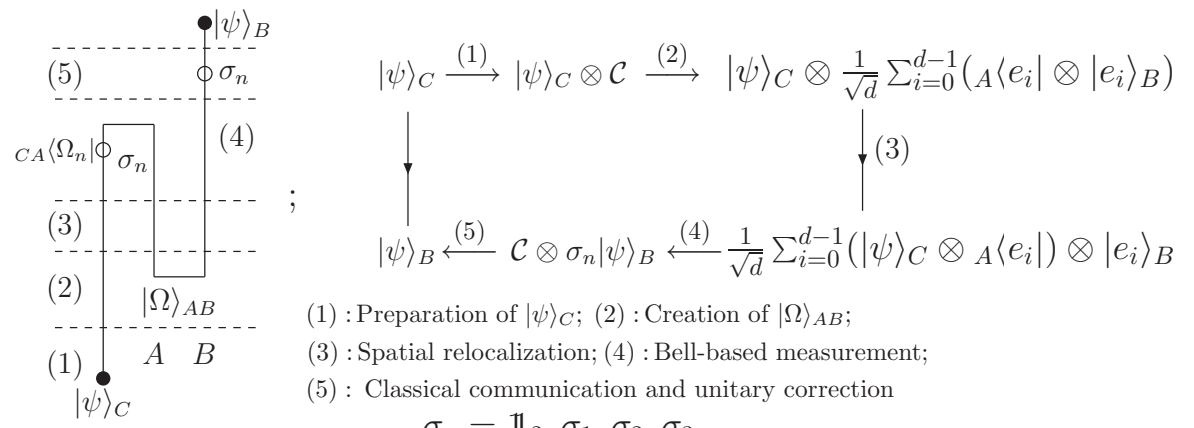

(1) : Preparation of $|\psi\rangle_{C} ;(2)$ : Creation of $|\Omega\rangle_{A B}$;

(3) : Spatial relocalization; (4) : Bell-based measurement;

(5): Classical communication and unitary correction

$$
\sigma_{n}=\mathbb{1}_{2}, \sigma_{1}, \sigma_{2}, \sigma_{3}
$$

Figure 26: Quantum information flow and strongly compact closed category.

theory in an intuitive way. But we will not involve mathematical details about the category theory since they have been treated in various ways in a series of papers by Abramsky, Coecke and Duncan, see [30, 42, 43, 44, 45, 46, 47, 48,

To transport Charlie's unknown quantum state $|\psi\rangle_{C}$ to Bob, the teleportation has to complete all the operations: preparation of $|\psi\rangle_{C}$; creation of $|\Omega\rangle_{A B}$ in Alice and Bob's systems; Bell-based measurement ${ }_{A B}\left\langle\Omega_{n}\right|$ in Charlie and Alice's systems; classical communication between Alice and Bob; local unitary correction by Bob. These steps divide the quantum information flow into six pieces which are shown up in the left diagrammatic term of Figure 26 where the third piece represents the process bringing Alice and Charlies' particles together for the entangling measurement. In the category theory, every step or piece is denoted by a specific map which satisfies the axioms of the strongly compact closed category theory. The crucial point is to identify the bijective correspondence between the Bell state and a map from the dual Hilbert space $\mathcal{H}^{*}$ to the Hilbert space $\mathcal{H}$, i.e.,

$$
\frac{1}{\sqrt{d}} \sum_{i=0}^{d-1}\left|e_{i}\right\rangle_{A} \otimes\left|e_{i}\right\rangle_{B} \approx \frac{1}{\sqrt{d}} \sum_{i=0}^{d-1}{ }_{A}\left\langle e_{i}|\otimes| e_{i}\right\rangle_{B}, \quad \mathcal{H}_{A} \otimes \mathcal{H}_{B} \approx \mathcal{H}_{A}^{*} \otimes \mathcal{H}_{B}
$$

so that the strongly compact closed category theory has a physical realization in the form of the quantum information flow, see the right diagrammatic term of Figure 26 where the symbol $\mathcal{C}$ denotes the complex field and we apply

$$
|\psi\rangle_{C} \approx|\psi\rangle_{C} \otimes \mathcal{C}, \quad|\psi\rangle_{B} \approx \mathcal{C} \otimes|\psi\rangle_{B}
$$

and create a bipartite state from a complex number $\mathcal{C}$ and also annihilate it into $\mathcal{C}$.

In the paper, we apply the Temperley-Lieb category to various topics around the teleportation and obtain nice pictures and useful formulas. It is obviously different from the strongly compact closed category. The essential conceptual differences have been listed clearly in the above subsection by comparing Figure 24 with Figure 25. Additionally, the appearance of $\mathcal{H}^{*} \otimes \mathcal{H}$ is not require by the teleportation or even 
the quantum information flow but is imposed by the axioms of the strongly compact closed category theory, see Figure 26. The quantum information flow (its description of the strongly compact closed category theory) is only a part of the Temperley-Lieb diagrammatic representation for the teleportation, as is observed by reading Figure 24, Figure 25 and Figure 26 together.

As a remark, the category theory [45, 48, 49, 50] is expected to play the roles in quantum information theory and will be involved in our forthcoming research.

\section{Concluding remarks}

In the paper, we introduce the braid group, the Temperley-Lieb algebra and the Brauer algebra to the mathematical description of the teleportation. Besides the proposals for the virtual braid teleportation and braid teleportation, We design diagrammatic rules for algebraic expressions in order to show the Temperley-Lieb algebra under local unitary transformations to underly the teleportation. Our diagrammatic approach has been applied to various topics around the teleportation: the transfer operator and acausality problem, teleportation and measurement, all tight teleportation and dense coding schemes, the Temperley-Lieb algebra and maximally entangled states; entanglement swapping; teleportation and topological quantum computing; teleportation and the Brauer algebra; multipartite entanglements. A crucial point is to recognize the teleportation configuration to be a fundamental element of the Brauer algebra which suggests an equivalent realization of the teleportation in terms of the swap gate and Bell measurements. Also, we compare our diagrammatic approach with the teleportation topology and categorical descriptions for the quantum information flow so that those essential differences among them are made clear.

As concluding remarks, in this paper we do not intend to bring new physics or new mathematics to the field of quantum information. We strongly believe in the existence of beautiful mathematical structures behind entanglement and teleportation such as the braid group and Temperley-Lieb algebra which are well known to the community of knot theory for a long time. They not only simplify complicated algebraic calculation in an intuitive diagrammatic way but also oblige us to accept that quantum phenomena like entanglement and teleportation should be no mysterious at all for the mankind living in the classical world once we are equipped with suitable mathematical tools. As Abramsky and Coecke suggested [51, we are also looking for a powerful mathematical framework to describe various topics of quantum information in a natural and unified way which has to be beyond the von Neumman formalism for standard quantum mechanics.

\section{Acknowledgements}

I am indebted to X.Y. Li for his constant encouragements and supports, and grateful to M.L. Ge, L.H. Kauffman and R.F. Werner for their helpful comments on 
Yang-Baxter equations, teleportation topology and tight teleportation schemes, respectively. I especially thank R.F. Werner for his email correspondence from Dresden on the project of setting up a bridge between knot theory and quantum information. This work is in part supported by NSFC-10447134 and SRF for ROCS, SEM.

\section{References}

[1] R.F. Werner, Quantum States with Einstein-Podolsky-Rosen Correlations Admitting a Hidden-Variable Model, Phys. Rev. A 40 (1989) 4277.

[2] M. Nielsen and I. Chuang, Quantum Computation and Quantum Information (Cambridge University Press, 1999).

[3] P.W. Shor, Polynomial Time Algorithms for Prime Factorization and Discrete Logrithms on a Quantum Computer.

[4] L.K. Grover, Quantum Mechanics Helps in Searching for a Needle in a Haystack, Phys. Rev. Lett. 78 (1997) 325-328.

[5] C.H. Bennett, G. Brassard, Quantum Cryptography: Public Key Distribution and Coin Tossing, Int. Conf. Computers, Systems \& Signal Processing, Bangalore, India, December 10-12, 1984, pp. 175-179.

[6] A.K. Ekert, Quantum Cryptography Based on Bell's Theorem, Phys. Rev. Lett. 67 (1991) 661-663.

[7] C.H. Bennett, G. Brassard, C. Crepeau, R. Jozsa, A. Peres and W. K. Wootters, Teleporting an unknown quantum state via dual classical and Einstein-PodolskyRosen channels, Phys. Rev. Lett. 70 (1993) 1895-1899.

[8] L.H. Kauffman, Knots and Physics (World Scientific Publishers, 2002).

[9] J.L. Brylinski and R. Brylinski, Universal quantum gates, in Mathematics of Quantum Computation, Chapman \& Hall/CRC Press, Boca Raton, Florida, 2002 (edited by R. Brylinski and G. Chen).

[10] L.H. Kauffman and S.J. Lomonaco Jr., Braiding Operators are Universal Quantum Gates, New J. Phys. 6 (2004) 134. Arxiv: quant-ph/0401090.

[11] Y. Zhang, L.H. Kauffman and M.L. Ge, Universal Quantum Gate, YangBaxterization and Hamiltonian. Int. J. Quant. Inform., Vol. 3, 4 (2005) 669-678. Arxiv: quant-ph/0412095

[12] Y. Zhang, L.H. Kauffman and M.L. Ge, Yang-Baxterizations, Universal Quantum Gates and Hamiltonians. Quant. Inf. Proc. 4 (2005) 159-197. Arxiv: quant-ph/0502015. 
[13] C.N. Yang, Some Exact Results for the Many Body Problems in One Dimension with Repulsive Delta Function Interaction, Phys. Rev. Lett. 19 (1967) 13121314.

[14] R.J. Baxter, Partition Function of the Eight-Vertex Lattice Model, Annals Phys. 70 (1972) 193-228.

[15] L.H. Kauffman, Quantum Computation and the Jones Polynomial, in Quantum Computation and Information, S. Lomonaco, Jr. (ed.), AMS CONM/305, 2002, pp. 101-137. Arxiv: math. QA/0105255.

[16] L.H. Kauffman, Quantum Topology and Quantum Computing, in Quantum Computation, S. Lomonaco (ed.), AMS PSAPM/58, 2002, pp. 273-303.

[17] L. H. Kauffman and S. J. Lomonaco Jr., Quantum Knots, in E. Donkor, A.R. Pirich and H.E. Brandt (eds.), Quantum Information and Computation II, Spie Proceedings, (12 -14 April, Orlando, FL, 2004), Vol. 5436, pp. 268-284. Arxiv: quant-ph/0403228.

[18] L.H. Kauffman and S.J. Lomonaco Jr., Quantum Entanglement and Topological Entanglement, New J. Phys. 4 (2002) 73.1-73.18.

[19] L.H. Kauffman and S.J. Lomonaco Jr., Entanglement Criteria-Quantum and Topological, in E. Donkor, A.R. Pirich and H.E. Brandt (eds.), Quantum Information and Computation - Spie Proceedings, (21-22 April, Orlando, FL, 2003), Vol. 5105, pp. 51-58. Arxiv: quan-ph/0304091

[20] L.H. Kauffman, Teleportation Topology. Opt. Spectrosc. 9 (2005) 227-232. Arxiv: quan-ph/0407224.

[21] R. F. Werner, All Teleportation and Dense Coding Schemes, JPA. Arxiv: quant-ph/0003070.

[22] Y. Zhang, L.H. Kauffman and R.F. Werner, Permutation and its Partial Transpose (in preparation).

[23] H.N.V. Temperley and E.H. Lieb, Relations between the 'Percolation' and 'Colouring' Problem and Other Graph-Theoretical Problems Associated with Regular Planar Lattices: Some Exact Results for the 'Percolation' Problem, Proc. Roy. Soc. A 322 (1971) 251-280.

[24] L.H. Kauffman, Virtual Knot Theory, European J. Comb. 20 (1999) 663-690.

[25] R. Brauer, On Algebras Which Are Connected With the Semisimple Continuous Groups, Ann. of Math. 38 (1937) 857-872.

[26] L. Vaidman, Teleportation of Quantum States, Phys. Rev. A 49 (1992) 14731475. 
[27] J. Preskill, Quantum Information and Computation, Lecture Notes for Ph219/CS219, Chapter 4, pp. 26-35.

[28] M. Żukowski, A. Zeilinger, M.A. Horne and A.K. Ekert, 'Event-ready-detectors' Bell experiment via entanglement swapping. Phys. Rev. Let. 71 (1993) 42874290.

[29] D. Gottesman and I.L. Chuang, Quantum teleportation is a universal computational primitive. Nature 402 (1999) 390-393. Arxiv:quant-ph/9908010.

[30] B. Coecke, The logic of entanglement. An invitation. Oxford University Computing Laboratory Research Report nr. PRG-RR-03-12. An 8 page short version is at Arxiv:quant-ph/0402014. The full 160 page version is at web.comlab.ox.ac.uk/oucl/ publications/tr/rr-03-12.html.

[31] H.A. Dye, Unitary Solutions to the Yang-Baxter Equation in Dimension Four, Quant. Inf. Proc. 2 (2003) 117-150. Arxiv: quant-ph/0211050

[32] Y. Zhang, N.H. Jing and M.L. Ge, New Quantum Algebras via RRT Relation on Eight-Vertex Models, (in preparation).

[33] J. Franko, E.C. Rowell and Z.H. Wang, Extraspecial 2-groups and images of braid group representations. Arxiv: math.RT/0503435

[34] F. Wilczek, Fractional Statistics and Anyon Superconductivity (World Scientific, 1990).

[35] N. Erez, Teleportation from a Projection Operator Point of View. Arxiv: quant-ph/0510130

[36] L.H. Kauffman and S.L. Lin, Temperley-Lieb Recoupling Theory and Invariants of Three-Manifold, Ann. of Math. Stud. 114 (Princeton Univ. Press, 1994).

[37] P.W. Shor, Fault-tolerant Quantum Computation. In Proceedings, 35th Annual Symposium on Fundamentals of Computer Science (IEEE Press, Los Alamitos, 1996) 56-65. Arxiv: quant-ph/9605011

[38] J. Preskill, Fault-tolerant Quantum Computation. Arxiv: quant-ph/9712048.

[39] A. Yu. Kitaev, Fault-tolerant Quantum Computation by Anyons, Annals Phys. 303 (2003) 2-30. Arxiv: quant-ph/9707021.

[40] M.H. Freedman, M.J. Larsen and Z. Wang, The Two-Eigenvalue Problem and Density of Jones Representation of Braid Groups, Comm. Math. Phys. 228 (2002) 177-199.

[41] D. Aharonov, V. Jones and Z. Landau, A Polynomial Quantum Algorithm for Approximating the Jones Polynomial. Arxiv: quant-ph/0511096. 
[42] S. Abramsky, and B. Coecke, Physical traces: quantum vs. classical information processing. Electronic notes on Theoretical Computer Science 69 (special issue: Proceedings of Category Theory in Computer Science 2002). Arxiv:cs/0207057

[43] S. Abramsky, and B. Coecke, Abstract physical traces. Theory and Applications of Categories 14 (2003) 111-124.

[44] B. Coecke, Quantum information-flow, concretely, and axiomatically. In: Proceedings of Quantum Informatics 2004, pp. 15-29, Y. I. Ozhigov, Ed., Proceedings of SPIE Vol. 5833. Arxiv:quant-ph/0506132.

[45] S. Abramsky, and B. Coecke, A categorical semantics of quantum protocols. In: Proceedings of the 19th Annual IEEE Symposium on Logic in Computer Science (LiCS`04), IEEE Computer Science Press. Arxiv:quant-ph/0402130

[46] B. Coecke, De-linearizing linearity: Projective quantum axiomatics from strong compact closure. Electronic Notes in Theoretical Computer Science (special issue: Proceedings of the 3rd International Workshop on Quantum Programming Languages). Arxiv:quant-ph/0506134.

[47] B. Coecke, Kindergarten Quantum Mechanic. Arxiv: quant-ph/0510032.

[48] S. Abramsky and R. Duncan, A Categorical Quantum Logic. Arxiv: quant-ph/0512114.

[49] J. Baez, Quantum quandaries: a category-theoretic perspective. In: S. French et al. (Eds.) Structural Foundations of Quantum Gravity, Oxford University Press. Arxiv:quant-ph/0404040

[50] P. Selinger, Dagger compact closed categories and completely positive maps. Electronic Notes in Theoretical Computer Science (special issue: Proceedings of the 3rd International Workshop on Quantum Programming Languages).

[51] S. Abramsky, High-level Methods for Quantum Computation and Quantum Information. Pleanary talk at 19th Annual IEEE Symposium on Logic in Computer Science (LiCS'04). Proceedings of the 19th Annual IEEE Symposium on Logic in Computer Science, IEEE Computer Science Press. 